\title{
Multi-Clock Timed Networks
}

\author{
Parosh Aziz Abdulla, Johann Deneux, and Pritha Mahata \\ Dept. of Information Technology \\ Uppsala University \\ Sweden \\ \{parosh, johannd, pritha\}eit.uu.se
}

\begin{abstract}
We consider verification of safety properties for parameterized systems of timed processes, so called timed networks. A timed network consists of a finite state process, called a controller, and an arbitrary set of identical timed processes. In a previous work, we showed that checking safety properties is decidable in the case where each timed process is equipped with a single real-valued clock. It was left open whether the result could be extended to multi-clock timed networks. We show that the problem becomes undecidable when each timed process has two clocks.

On the other hand, we show that the problem is decidable when clocks range over a discrete time domain. This decidability result holds when processes have any finite number of clocks.
\end{abstract}

\section{Introduction}

One of the main current challenges in model checking is to extend its applicability to parameterized systems. The description of such a system is parameterized by the number of components, and the challenge is to check correctness of all instances in one verifi cation step. Most existing methods for model checking of parameterized systems consider the case where each individual component is modelled as a fi nite-state process.

In this paper we study parameterized systems of timed processes, so called Timed Networks (TNS). A TN represents a family of systems, each consisting of a fi nite-state controller, together with fi nitely, but arbitrarily many timed processes (timed automata). A timed process operates on a fi nite number of real-valued clocks. This means that a $\mathrm{TN}$ operates on an unbounded number of clocks, and therefore its behaviour cannot be captured by that of a timed automaton [AD94].

In [AJ03], we show decidability of the controller state reachability problem for TNs: given a state of the controller, is there a computation from an initial confi guration leading to that state? This problem is relevant since it can be shown, using standard techniques, that checking large classes of safety properties can be reduced to controller state reachability. The decidability result in [AJ03] is given subject to the restriction that each timed process has a single clock. As an example, this allows automatic verifi cation of a parameterized version of Fischer's protocol (see e.g. [KLL ${ }^{+97]) . ~ T h i s ~ p r o t o c o l ~}$ achieves mutual exclusion by defi ning timing constraints on an arbitrary set of processes each with one clock. We can show correctness of the protocol regardless of the number of participating processes. The paper [AJ03] leaves open the case of multi-clock TNs, i.e., TNs where each timed process may have several clocks. 
In the literature, there are many applications where a number of timed automata [AD94] run in parallel and where each of the timed automata has more than one clock. For instance, the Phillips audio control protocol with bus collision [BGK $\left.{ }^{+} 96\right]$ has two clocks per sender of audio signals. Also, the system described in [MT01] consists of an arbitrary number of nodes, each of which is connected to a set of LANs. Each node maintains timers to keep track of sending and receiving of messages from other nodes connected to the same set of LANs. In a similar way to Fischer's protocol, it is clearly relevant to ask whether we can verify correctness of the protocol in $\left[\mathrm{BGK}^{+} 96\right]$ regardless of the number of senders, or the protocol in [MT01] regardless of the number of nodes.

The question is then whether the decidability result of [AJ03] can be extended to multi-clock systems. In this paper we answer this question negatively. In fact, we show that it is suffi cient to allow two clocks per process in order to get undecidability. The undecidability result is shown through a reduction from the classical reachability problem for 2-counter machines. The main ingredient in the undecidability proof is an encoding of counters which allows testing for zero. The encoding represents each counter by a linked list of processes, where ordering on elements of the list is reflected by ordering on clock values of the relevant processes, and where the link between two elements in the list is encoded by whether two clocks belong to the same process. The value of a counter is reflected by the length of the corresponding list.

We also consider Discrete Timed Networks (DTNs): a variant of timed networks where clocks are interpreted over a discrete time domain rather than a dense one. Surprisingly, it turns out that the controller state reachability problem now becomes decidable. The decidability result holds regardless of the number of clocks allowed inside each timed process. We show decidability using the theory introduced in [AČJYK00] for verifi cation of transition systems which are monotonic with respect to a well quasiordering. More precisely, we defi ne a counter abstraction for DTNs. This is an exact abstraction of the system where we only count the number of processes which have certain states and certain clock values. We show that such an abstraction induces a well quasi-ordering, and that the behaviour of a DTN is monotonic with respect to that ordering.

Related Work Most works on verifi cation of parameterized systems consider the case where each component is a finite-state system. Applications include cache coherence protocols [Del00,EK03], broadcast protocols [EFM99], mutual exclusion protocols with linear topologies $\left[\mathrm{KMM}^{+} 01\right]$, etc.

In [AMC02] a method is given for translating a timed automaton with several clocks into the parallel composition of a fi nite number of automata each operating on a single clock. This may give the impression that reachability problems for multi-clock TNs can in a similar way be reduced to corresponding problems for single-clock TNs. However, the construction given in [AMC02] will not work in our case. The reason is that, due to the unbounded number of timed processes, it is not possible to keep track of clocks belonging to the same process.

The works in [AAB00,AHV93] consider timed automata which are parameterized in the the following sense: transitions are guarded with predicates which compare clocks (and counters) with parameters possibly ranging over infi nite domains. The models used 
in these papers assume a fi nite number of clocks and are therefore orthogonal to the models considered in this paper.

A work related to our result on DTNs is [GS92] where counter abstraction is used to obtain a Petri net model for parameterized systems. However, a process in [GS92] is assumed to be fi nite-state. Furthermore, counter abstraction in the case of DTNs yields a model with a different behaviour than that of Petri nets.

Outline: Section 2 gives the defi nition of timed networks. Section 3 recalls the classical model of 2-counter machines. Section 4 shows how a confi guration of a 2-counter machine can be encoded by a confi guration of a timed network, while Section 5 shows how the transitions of a 2-counter machine can be simulated by transitions of a timed network. We give the correctness proof for our encoding in Section 6. In Section 7 we give an algorithm for deciding the controller-state reachability problem for DTNs. Finally in Section 8, we conclude and give some directions for future work.

\section{Definitions}

In this section, we defi ne timed networks: families of (infi nitely many) systems each consisting of a controller and an arbitrary number of identical timed processes. The controller is a fi nite state automaton while each process is a timed automaton [AD94], i.e., a fi nite-state automaton which operates on a fi nite number of local real-valued clocks $x_{1}, \ldots, x_{K}$. The values of all clocks are incremented continuously at the same rate. In addition, the network can change its confi guration according to a fi nite number of rules. Each rule describes a set of transitions in which the controller and a fi xed number of processes synchronize and simultaneously change their states. A rule may be conditioned on the local state of the controller, together with the local states and clock values of the processes. If the conditions for a rule are satisfi ed, then a transition may be performed where the controller and each participating process changes its state. Also, during a transition, a process may reset some of its clocks to 0 .

We use $\mathbb{N}$ and $\mathbb{R}^{\geq 0}$ for the set of natural numbers and set of non-negative real numbers respectively.

Timed Networks A family of timed networks (timed network for short) $\mathcal{N}$ with $K$ clocks is a pair $(Q, \mathcal{R})$, where:

- $Q$ is a fi nite set of states. The set $Q$ is the union of two disjoint sets; the set $Q^{c t r l}$ of controller states, and the set $Q^{\text {proc }}$ of process states. These sets contain two distinguished initial (idle) states, namely $i d l e^{c} \in Q^{\text {ctrl }}$ and $i d l e^{p} \in Q^{\text {proc }}$.

- $\mathcal{R}$ is a fi nite set of rules where each rule is of the form

$$
\left[\begin{array}{c}
q_{0} \\
\overrightarrow{q_{0}^{\prime}}
\end{array}\right]\left[\begin{array}{c}
q_{1} \\
g_{1} \rightarrow R_{1} \\
q_{1}^{\prime}
\end{array}\right] \quad \cdots \quad\left[\begin{array}{c}
q_{n} \\
g_{n} \rightarrow R_{n} \\
q_{n}^{\prime}
\end{array}\right]
$$

such that $q_{0}, q_{0}^{\prime} \in Q^{\text {ctrl }}$, and for all $i: 1 \leq i \leq n$ we have: $q_{i}, q_{i}^{\prime} \in Q^{\text {proc }}$, and $g_{i} \rightarrow R_{i}$ is a guarded command where $g_{i}$ is a boolean combination of predicates of the form $k \triangleright x$ for $k \in \mathbb{N}, \triangleright \in\{=,<, \leq,>, \geq\}, x \in\left\{x_{1}, \ldots, x_{K}\right\}$ and $R_{i} \subseteq\left\{x_{1}, \ldots, x_{K}\right\}$. 
Intuitively, the set $Q^{\text {ctrl }}$ represents the states of the controller and the set $Q^{\text {proc }}$ represents the states of the processes. A rule of the above form describes a set of transitions of the network. The rule is enabled if the state of the controller is $q_{0}$ and if there are $n$ processes with states $q_{1}, \cdots, q_{n}$ whose clock values satisfy the corresponding guards. The rule is executed by simultaneously changing the state of the controller to $q_{0}^{\prime}$ and the states of the $n$ processes to $q_{1}^{\prime}, \cdots, q_{n}^{\prime}$, and resetting the clocks belonging to the sets $R_{1}, \ldots, R_{n}$.

For a guard $g_{i}$ we write $g_{i}\left(y_{1}, \ldots, y_{K}\right)$ to denote the Boolean expression which results from substituting the occurrences of $x_{1}, \ldots, x_{K}$ in $g_{i}$ by $y_{1}, \ldots, y_{K}$ respectively.

Configurations A confi guration $\gamma$ of a timed network $(Q, \mathcal{R})$ with $K$ clocks is a tuple of the form $(I, q, \mathcal{Q}, X)$, where $I$ is a fi nite index set, $q \in Q^{\text {ctrl }}, \mathcal{Q}: I \rightarrow Q^{\text {proc }}$, and $X:\{1, \ldots, K\} \rightarrow I \rightarrow \mathbb{R}^{\geq 0}$.

Intuitively, the confi guration $\gamma$ refers to the controller whose state is $q$, and to $|I|$ processes, whose states are defi ned by $\mathcal{Q}$. The clock values of the processes are defi ned by $X$. More precisely, for $k: 1 \leq k \leq K$ and $i \in I, X(k)(i)$ gives the value of clock $x_{k}$ in the process with index $i$.

We use $|\gamma|$ to denote the number of processes in $\gamma$, i.e., $|\gamma|=|I|$. Also, we shall use $X_{k}$ to denote the mapping $I \rightarrow \mathbb{R}^{\geq 0}$ such that $X_{k}(i)=X(k)(i)$.

Example 1. Figure 1 shows graphical representation of a confi guration in a timed network with two clocks, given by $(\{1,2,3\}, q, \mathcal{Q}, X)$ where $\mathcal{Q}(1)=q_{1}, \mathcal{Q}(2)=$ $q_{2}, \mathcal{Q}(3)=q_{3}$ and $X_{1}(1)=0.1, X_{1}(2)=0.5, X_{1}(3)=5.0, X_{2}(1)=2.3, X_{2}(2)=$ $1.4, X_{2}(3)=0.6$.

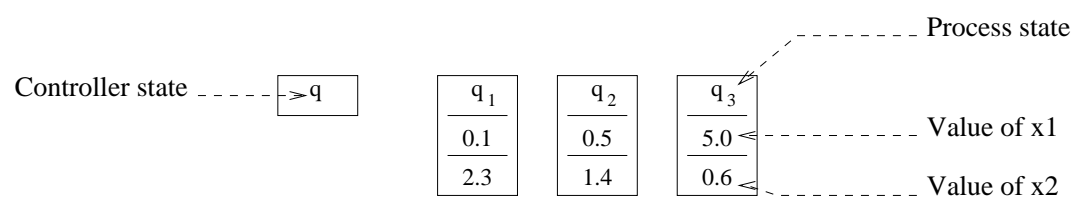

Fig. 1. Graphical representation of a configuration in a timed network with two clocks.

Transition Relation The timed network $\mathcal{N}$ above induces a transition relation $\longrightarrow$ on the set of confi gurations. The relation $\longrightarrow$ is the union of a discrete transition relation $\longrightarrow_{D}$, representing transitions induced by the rules, and a timed transition relation $\longrightarrow_{T}$ which represents passage of time.

The discrete relation $\longrightarrow_{D}$ is the union $\bigcup_{r \in \mathcal{R}} \longrightarrow_{r}$, where $\longrightarrow_{r}$ represents a transition performed according to rule $r$. Let $r$ be a rule of the form described in the above defi nition of timed networks. Consider two confi gurations $\gamma=(I, q, \mathcal{Q}, X)$ and $\gamma^{\prime}=\left(I, q^{\prime}, \mathcal{Q}^{\prime}, X^{\prime}\right)$. We use $\gamma \rightarrow_{r} \gamma^{\prime}$ to denote that there is an injection $h:\{1, \ldots, n\} \rightarrow I$ such that for each $i: 1 \leq i \leq n$ and $k: 1 \leq k \leq K$ we have:

1. $q=q_{0}, \mathcal{Q}(h(i))=q_{i}$, and $g_{i}\left(X_{1}(h(i)), \ldots, X_{K}(h(i))\right)$ holds. That is, the rule $r$ is enabled. 
2. $q^{\prime}=q_{0}^{\prime}$, and $\mathcal{Q}^{\prime}(h(i))=q_{i}^{\prime}$. The states are changed according to $r$.

3. If $x_{k} \in R_{i}$ then $X_{k}^{\prime}(h(i))=0$, while if $x_{k} \notin R_{i}$ then $X_{k}^{\prime}(h(i))=X_{k}(h(i))$. In other words, a clock is reset to 0 if it occurs in the corresponding set $R_{i}$. Otherwise its value remains unchanged.

4. $\mathcal{Q}^{\prime}(j)=\mathcal{Q}(j)$ and $X_{k}^{\prime}(j)=X_{k}(j)$, for $j \in I \backslash \operatorname{range}(h)$, i.e., the process states and the clock values of the non-participating processes remain unchanged.

For a confi guration $\gamma=(I, q, \mathcal{Q}, X)$ and $t \in \mathbb{R}^{\geq 0}$, we use $\gamma^{+t}$ to denote the confi guration $\left(I, q, \mathcal{Q}, X^{\prime}\right)$ where $X_{k}^{\prime}(j)=X_{k}(j)+t$ for each $j \in I$ and $k: 1 \leq k \leq K$. A timed transition is of the form $\gamma \longrightarrow_{T=t} \gamma^{\prime}$ where $\gamma^{\prime}=\gamma^{+t}$. Such a transition lets time pass by $t$. We use $\gamma \longrightarrow_{T} \gamma^{\prime}$ to denote that $\gamma \longrightarrow_{T=t} \gamma^{\prime}$ for some $t \in \mathbb{R}^{\geq 0}$.

We defi ne $\longrightarrow$ to be $\longrightarrow_{D} \cup \longrightarrow_{T}$ and use ${ }^{*} \rightarrow$ to denote the reflexive transitive closure of $\longrightarrow$. Notice that if $\gamma \longrightarrow \gamma^{\prime}$ then the index sets of $\gamma$ and $\gamma^{\prime}$ are identical and therefore $|\gamma|=\left|\gamma^{\prime}\right|$. For a confi guration $\gamma$ and a controller state $q$, we use $\gamma \stackrel{*}{\longrightarrow} q$ to denote that there is a confi guration $\gamma^{\prime}$ of the form $\left(I^{\prime}, q^{\prime}, \mathcal{Q}^{\prime}, X^{\prime}\right)$ such that $\gamma \stackrel{*}{\longrightarrow} \gamma^{\prime}$ and $q^{\prime}=q$.

Reachability A confi guration $\gamma_{i n i t}=(I, q, \mathcal{Q}, X)$ is said to be initial if $q=i d l e^{c}$, $\mathcal{Q}(i)=i d l e^{p}$, and $X_{k}(i)=0$ for each $i \in I$ and $k: 1 \leq k \leq K$. This means that an execution of a timed network starts from a confi guration where the controller and all the processes are in their initial states, and the clock values are all equal to 0 . Notice that there is an infi nite number of initial confi gurations, namely one for each index set $I$.

\section{Controller State Reachability Problem $(\mathrm{TN}(K)$-Reach)}

Instance A timed network $(Q, \mathcal{R})$ with $K$ clocks and a controller state $q_{F}$.

Question Is there an initial confi guration $\gamma_{\text {init }}$ such that $\gamma_{\text {init }} \stackrel{*}{\longrightarrow} q_{F}$ ?

Controller state reachability is relevant, since it can be shown, using standard techniques [VW86,GW93], that checking safety properties (expressed as regular languages) can be translated into instances of the problem. In [AJ03] we show that TN(1)-Reach is decidable. In this paper we show

Theorem 1. TN(2)-Reach is undecidable.

\section{2-Counter Machines}

In this section we recall the standard defi nition of counter machines. Here, we assume that such a machine operates on two counters which we call $c_{1}$ and $c_{2}$.

A two-counter machine $\mathcal{C}$ is a tuple $(S, \mathcal{I})$ where $S$ is a fi nite set of local states with a distinguished initial local state $s_{\text {init }} \in S$, and $\mathcal{I}$ is a fi nite set of instructions. An instruction $\theta$ is a triple $\left(s_{1}, o p, s_{2}\right)$, where $s_{1}, s_{2} \in S$ and $o p$ is either an increment (of the form $c_{1}++$ or $c_{2}++$ ); a decrement (of the form $c_{1}--$ or $c_{2}--$ ); or a zero testing (of the form $c_{1}=0$ ? or $c_{2}=0$ ?). A configuration $\beta$ of a two-counter machine is a triple $\left(s, m_{1}, m_{2}\right)$, where $s \in S$ represents the local state, and $m_{1}, m_{2} \in \mathbb{N}$ represent the 
values of the counters $c_{1}$ and $c_{2}$ respectively. The counter machine $\mathcal{C}$ induces a transition relation $\leadsto$ on the set of confi gurations, which is defi ned as usual using the standard interpretations of counter operations. We use $\stackrel{*}{\sim}$ to denote the reflexive transitive closure of $\leadsto$. In a similar manner to timed networks, we use $\beta \stackrel{*}{\sim} s$ to denote that there is a confi guration $\beta=\left(s^{\prime}, m_{1}^{\prime}, m_{2}^{\prime}\right)$ such that $\beta \stackrel{*}{\sim} \beta^{\prime}$ and $s^{\prime}=s$. We defi ne the initial configuration $\beta_{\text {init }}$ to be $\left(s_{\text {init }}, 0,0\right)$. The control state reachability problem for a 2 counter machines (CM-Reach) is: given local state $s_{F}$ check whether $\beta_{i n i t} \stackrel{*}{\sim} s_{F}$. The following result [Min61] is well-known.

Theorem 2. CM-Reach is undecidable.

In our correctness proof (Section 6), we use the relation $\stackrel{n}{\sim}$, with $n \geq 0$, on confi gurations, where $\beta \stackrel{n}{\sim} \beta^{\prime}$ iff there is a sequence $\beta_{0} \leadsto \beta_{1} \leadsto \cdots \leadsto \beta_{n}$ with $\beta_{0}=\beta$ and $\beta_{n}=\beta^{\prime}$. The relation $\stackrel{n}{\sim}$ is extended to local states in a similar manner to $\stackrel{*}{\rightarrow}$. Notice that $\stackrel{*}{\sim}=\cup_{n} \stackrel{n}{\sim}$.

\section{Encoding of Configurations}

We show undecidability of TN(2)-Reach through a reduction from CM-Reach. Given a counter machine $\mathcal{C}=(S, \mathcal{I})$, we shall derive a timed network $\mathcal{N}_{\mathcal{C}}=\left(Q_{\mathcal{C}}, \mathcal{R}_{\mathcal{C}}\right)$ with two clocks. In this section, we perform the fi rst step in the reduction; namely we describe how to construct the set $Q_{\mathcal{C}}$. Also, we describe how confi gurations of $\mathcal{C}$ are encoded as confi gurations of $\mathcal{N}_{\mathcal{C}}$. Finally, we introduce a special type of encodings, called proper encodings, which we use in our simulation of $\mathcal{C}$.

States According to the model described in Section 2, the set $Q_{\mathcal{C}}$ will consist of two disjoint sets of states: the set $Q_{\mathcal{C}}^{c t r l}$ of controller states and the set $Q_{\mathcal{C}}^{\text {proc }}$ of process states. The set $Q_{\mathcal{C}}^{c t r l}$ contains three types of states:

1. The initial controller state $i d l e^{c}$.

2. Local states of $\mathcal{C}$ : all members of $S$ have copies in $Q_{\mathcal{C}}^{c t r l}$.

3. Temporary states: the set $Q_{\mathcal{C}}^{c t r l}$ contains a state $t m p^{\theta}$ for each increment instruction $\theta \in \mathcal{I}$. These states are used as intermediate states during the simulation of increments (Section 5). The set $Q_{\mathcal{C}}^{c t r l}$ also contains the state $s_{\text {init }}^{\prime}$ (recall that $s_{\text {init }}$ is the initial local state of $\mathcal{C}$ ). This state is used as an intermediate state in the initialization phase of the the simulation (Section 5).

The set $Q_{\mathcal{C}}^{\text {proc }}$ contains two types of states:

1. The initial process state $i d l e^{p}$.

2. Six states $f s t_{1}, m_{i} d_{1}$, last $_{1}, f s t_{2}, m_{i} d_{2}$, and last $_{2}$, used for encoding the two counters (as described below).

Encodings Each confi guration $\beta$ of $\mathcal{C}$ will be encoded by a set of confi gurations in $\mathcal{N}_{\mathcal{C}}$. The local state of $\beta$ will be encoded by the controller state. Each counter will be modelled by a counter encoding. A counter encoding arranges a set of processes as a circular list. The ordering among elements of the list is defi ned by the clock values. 
The length of the list reflects to the value of the counter. To defi ne counter encodings, we shall use the six process states $f s t_{1}$, mid $_{1}$, last $t_{1}$ (used for encoding of $c_{1}$ ), and $f s t_{2}$, mid $_{2}$, last $t_{2}$ (used for encoding of $c_{2}$ ). The states $f s t_{1}$ and last $_{1}$ are the states of the fir rst and last processes in the list encoding the value of $c_{1}$. All processes in the middle of the list will be in state $m_{i d_{1}}$. The states $f_{s t_{2}}$, mid $_{2}$, and last 2 play similar roles in the encoding of $c_{2}$. Formally, a confi guration $\gamma=(I, q, \mathcal{Q}, X)$ is said to be a $q$-encoding of value $m$ if there is an injection $h$ from the set $\{0, \ldots, m+1\}$ to $I$ such that the following conditions are satisfi ed

- $\mathcal{Q}(h(0))=f s t_{1}, \mathcal{Q}(h(m+1))=$ last $_{1}$, and $\mathcal{Q}(h(i))=$ mid $_{1}$ for each $i: 1 \leq i \leq$ $m$.

- $\mathcal{Q}(j) \in\left\{i d l e^{p}\right.$, fst $_{2}$, mid $_{2}$, last $\left._{2}\right\}$ if $j \in I \backslash \operatorname{range}(h)$.

- $X_{1}(h(i))<X_{1}(h(i+1))$ for each $i: 0 \leq i \leq m$.

- $X_{2}(h(i))=X_{1}(h((i+1) \bmod (m+2)))$, for each $i: 0 \leq i \leq m+1$.

The fir rst condition states that the processes which are part of a $q$-encoding are in one of the local states $f s t_{1}, m i d_{1}$, or last $t_{1}$. The second condition states that the processes which are not part of a $c_{1}$-encoding are in one of the local states $i d l e^{p}, f_{s t_{2}}$, mid $_{2}$, or last $_{2}$. The third and the fourth conditions show how the processes which are part of a $c_{1}$-encoding are ordered as a circular list. The position of each process in the list is reflected by values of its clocks $x_{1}$ and $x_{2}$. More precisely, the ordering among the $x_{1}$ clocks reflects the positions of the processes in the list. Also, clock $x_{2}$ of each process (except the last process) is equal to clock $x_{1}$ of the next process. Finally, clock $x_{2}$ of the last process is equal to clock $x_{1}$ of the fi rst process (giving the list a "circular" form). We use $\operatorname{Val}_{1}(\gamma)$ to denote the value $m$ of a $c_{1}$-encoding $\gamma$.

(a)

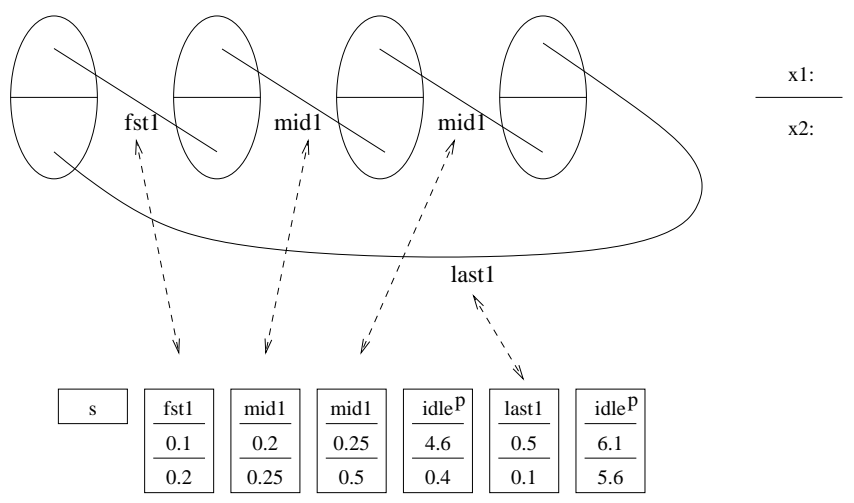

Fig. 2. (a) Graphical representation of ordering among clocks in $c_{1}$-encodings. (b) a $c_{1}$-encoding satisfying the ordering on clocks described in (a).

Example 2. Figure 2(b) shows a $c_{1}$-encoding of value 2. Figure 2(a) shows a graphical representation of the ordering among clock values. In Section 5 we shall use such a graphical representation to explain the different steps in the simulation of $\mathcal{C}$. Each ellipse 
contains clocks of equal values. Clocks in successive ellipses have increasing values, i.e., they are ordered from left (lower clock values) to right (higher clock values). The upper halves of the ellipses represent the $x_{1}$-clocks, while the lower halves represent the $x_{2}$-clocks. Each process is denoted by an edge whose end points are its two clocks. Such an edge is labelled by the current state of the process.

A $c_{2}$-encoding and its value $\operatorname{Val}_{2}(\gamma)$ are defi ned in a similar manner (replacing the states $f s t_{1}$, mid $_{1}$, and last $_{1}$ by $f s t_{2}$, mid $_{2}$, and last 2 in the encoding).

A confi guration $\gamma=(I, q, \mathcal{Q}, X)$ is said to be an encoding if the following two conditions are satisfi ed:

- $q=s$ for some $s \in S$, i.e., $q$ is the copy of a local state of $\mathcal{C}$.

- $\gamma$ is both a $c_{1}$ - and a $c_{2}$-encoding.

If $\gamma$ satisfi es the above conditions (i.e. if $\gamma$ is an encoding), we defi ne the signature $\operatorname{sig}(\gamma)$ of $\gamma$ to be the triple $\left(s, m_{1}, m_{2}\right)$, where $m_{1}=\operatorname{Val}_{1}(\gamma)$ and $m_{2}=\operatorname{Val}_{2}(\gamma)$. Intuitively, the triple $\left(s, m_{1}, m_{2}\right)$ will correspond to a confi guration of $\mathcal{C}$. Notice that several (in fact infi nitely many) confi gurations may have the same signature. However, all such confi gurations will have the same local states and the same orderings on clock values, and therefore will correspond to the same confi guration in $\mathcal{C}$.

Proper Encodings In our simulation of $\mathcal{C}$ we shall rely on a particular kind of encodings, called proper encodings. An encoding $\gamma$ of the form $(I, q, \mathcal{Q}, X)$ is said to be proper if it satisfi es the following condition:

- For each $i \in I$ with $\mathcal{Q}(i) \neq i d l e^{p}, 0<X_{1}(i), X_{2}(i)<1$.

In other words, all clocks participating in the encoding have values between (not including) zero and one. Certain steps of the simulation (see the decrementing operation in Section 5) are not possible to carry out without an upper bound on clock values of the processes. Working with proper encodings guarantees such an upper bound (namely an upper bound of one).

\section{Encoding of Transitions}

In this section, we perform the second step in deriving the timed network $\mathcal{N}_{\mathcal{C}}=$ $\left(Q_{\mathcal{C}}, \mathcal{R}_{\mathcal{C}}\right)$ from the counter machine $\mathcal{C}=(S, \mathcal{I})$. More precisely, we describe the set of rules $\mathcal{R}_{\mathcal{C}}$. The set $\mathcal{R}_{\mathcal{C}}$ contains the following rules:

Incrementing For each instruction $\theta=\left(s_{1}, c_{1}++, s_{2}\right)$ in $\mathcal{C}$ there are two rules in $\mathcal{R}_{\mathcal{C}}$, namely

$$
i n c_{1}^{\theta}:\left[\begin{array}{c}
s_{1} \\
\rightarrow \\
t m p^{\theta}
\end{array}\right]\left[\begin{array}{c}
f s t_{1} \\
0<x_{1} \rightarrow\left\{x_{1}\right\} \\
\text { mid }_{1}
\end{array}\right]\left[\begin{array}{c}
i d l e^{p} \\
\text { true } \rightarrow\left\{x_{2}\right\} \\
f s t_{1}
\end{array}\right]
$$

and the rule

$$
i n c_{2}^{\theta}:\left[\begin{array}{c}
t m p^{\theta} \\
\rightarrow \\
s_{2}
\end{array}\right]\left[\begin{array}{c}
f s t_{1} \\
0<x_{2} \rightarrow\left\{x_{1}\right\} \\
f s t_{1}
\end{array}\right]\left[\begin{array}{c}
\text { last }_{1} \\
{\text { true } \rightarrow\left\{x_{2}\right\}}^{\text {last }_{1}}
\end{array}\right]
$$


The total effect of the two rules is to increment the value of a $c_{1}$-encoding by adding one more process to the list. The rule $i n c_{1}^{\theta}$ changes the state of the process which is currently first in the list to mid . This process will be placed in the second position in the new encoding. At the same time a new process is picked from the set of idle processes, and its state is changed to $f_{s t} t_{1}$. The new process will be placed fi rst in the list. Furthermore, the rule resets (and therefore equates) clock $x_{1}$ and $x_{2}$ respectively of the two above mentioned processes. This is done in order to maintain the invariant that clock $x_{2}$ of each process (except the last process) is equal to clock $x_{1}$ of the next process (recall the defi nition of an encoding from Section 4 ). The result of applying rule $i n c_{1}^{\theta}$ on a $c_{1}$-encoding of value 2 is shown in Figure $3(\mathrm{~b})$.

Rule $i n c_{2}^{\theta}$ resets clock $x_{1}$ of the process which is now in state $f s t_{1}$ and clock $x_{2}$ of the process which is last in the list. This is done in order to maintain (i) the invariant that clock $x_{1}$ of a process (here the first process) is smaller than clock $x_{1}$ of the next process; and (ii) the invariant that clock $x_{2}$ of the last process is equal to clock $x_{1}$ of the fi rst process. The result of applying rule $i n c_{2}^{\theta}$ is shown in Figure 3(c).

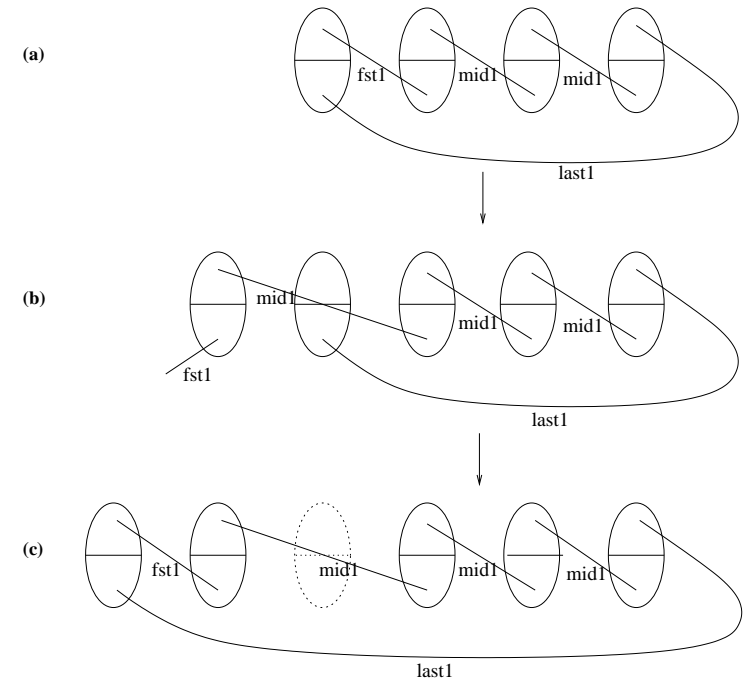

Fig. 3. Simulating $\left(s_{1}, c_{1}++, s_{2}\right)$ on a $c_{1}$-encoding

Some remarks about rules $i n c_{1}^{\theta}$ and $i n c_{2}^{\theta}$ :

- After execution of $i n c_{1}^{\theta}$, the controller will be in state $t m p^{\theta}$ and therefore $i n c_{2}^{\theta}$ is the only rule which may eventually be enabled after execution of $i n c_{1}^{\theta}$.

- The guard $0<x_{1}$ in the defi nition of $i n q_{1}^{\theta}$ is to guarantee that all clocks have positive values before the rule is applied. This makes sure that we avoid the scenario where we "accidentally" equate some clocks with the ones which are reset during the application of $i n c_{1}^{\theta}$. The same reasoning applies to the guard $0<x_{2}$ in the 
defi nition of the rule $i \mathrm{H}_{2}^{\theta}$. Similar guards exist in the rest of the rules described in this section.

- After application of $i n c_{2}^{\theta}$, the resulting encoding will not be proper, since clocks have just been reset and their values are now zero. We can re-create a proper encoding by letting time pass through a timed transition. Again, a similar reasoning is applicable to the rest of the rules described in this section.

Also, for each instruction of the form $\left(s_{1}, c_{2}++, s_{2}\right)$, there are two rules similar to the rules described above (replacing the states $f s t_{1}$, mid $_{1}$, and last $_{1}$ by $f_{s t_{2}}$, mid $_{2}$ and last $_{2}$, respectively).

Decrementing For each instruction $\theta=\left(s_{1}, c_{1}--, s_{2}\right)$ in $\mathcal{C}$ there is a rule in $\mathcal{R}_{\mathcal{C}}$, namely

$$
\operatorname{dec}^{\theta}:\left[\begin{array}{c}
s_{1} \\
\rightarrow \\
s_{2}
\end{array}\right]\left[\begin{array}{c}
\text { last }_{1} \\
x_{1}=1 \rightarrow \emptyset \\
\text { idle }^{p}
\end{array}\right]\left[\begin{array}{c}
f_{s t_{1}} \\
0<x_{1} \rightarrow\left\{x_{1}\right\} \\
f_{s t_{1}}
\end{array}\right]\left[\begin{array}{c}
\text { mid }_{1} \\
x_{2}=1 \rightarrow\left\{x_{2}\right\} \\
\text { last }_{1}
\end{array}\right]
$$

The rule $d e c^{\theta}$ decrements the value of a $c_{1}$-encoding by removing the last process of the list. More precisely, it changes the state of the last process to $i d l e^{p}$ (i.e. removes that process from the list), and changes the state of the process which is next last from mid $_{1}$ to last $_{1}$. In order to do that, we have to be able to identify the process which is next last in the list. Since all processes in the middle of the list are in state mid $_{1}$, we cannot identify the next last process simply by checking process states. Instead, we wait until the value of clock $x_{1}$ of the last process is equal to one. At that point of time, the process with clock $x_{2}$ equal to one is the next last process. Also, the rule resets (and therefore equates) clock $x_{1}$ of the fi rst process and clock $x_{2}$ of the next last process (which will now become last in the list). Figure 4 shows the effect of applying the rule to a $c_{1}$-encoding.

(a)

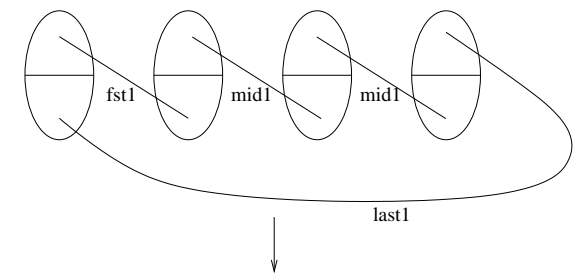

(b)

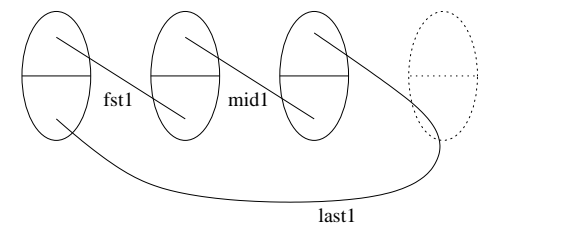

Fig. 4. Simulating $\left(s_{1}, c_{1}--, s_{2}\right)$ on a $c_{1}$-encoding 
Some remarks about the rule $d e c^{\theta}$ :

- Identifying the next last process (by waiting until some clocks are equal to one) uses the assumption that we start from a proper encoding. This implies that clocks of processes participating in the encoding have all values which are less than one. If this property is violated then the rule is not enabled (and will not become enabled through passage of time).

- The rule is not enabled in case the value of the $c_{1}$-encoding is equal to zero, since there will be no processes in state $m i d_{1}$.

- Waiting for clock $x_{1}$ of the last process in the $c_{1}$-encoding to become equal to one may enforce clocks of processes in the $c_{2}$-encoding to become greater than one. More precisely, this happens if some clock in a process which is part of the $c_{2}$ encoding has a greater value than clock $x_{1}$ of the process which is currently in state last $_{1}$. After applying $\operatorname{dec}^{\theta}$, the value of such clocks will be greater than one, and therefore the resulting confi guration will not be a proper encoding.

Figure 5 illustrates this scenario. We consider a proper encoding (shown in Figure $5(\mathrm{a}))$ with signature $\left(s_{1}, 1,1\right)$ such that clock $x_{1}$ of the process in state last $_{1}$ $(0.35)$ is smaller than that of the process in state last $_{2}(0.65)$. In order to enable the rule $d e c^{\theta}$, we let time pass until clock $x_{1}$ of the process last $_{1}$ becomes equal to one (shown in Figure 5(b)). However, at this point of time, both clock $x_{2}$ of a process in state mid $_{2}$ and $x_{1}$ of the process in state last $_{2}$ have become larger than one (1.3). Therefore, after applying the $d e c^{\theta}$, we get an encoding (of value $\left(s_{2}, 0,1\right)$ ) shown in Figure 5(c), which is not proper. This prevents any later application of decrementing and zero-testing rules.

In order, to maintain the possibility of maintaining proper encodings in our simulation, we combine the rule $d e c^{\theta}$ with the rotation rules described below.

(a)

\begin{tabular}{|c|c|c|c|c|c|c|}
\hline s1 & fst1 & mid1 & last1 & fst2 & mid2 & last2 \\
\hline & 0.2 & 0.3 & 0.35 & 0.1 & 0.25 & 0.65 \\
\hline & 0.3 & 0.35 & 0.2 & 0.25 & 0.65 & 0.1 \\
\hline
\end{tabular}

(b)

\begin{tabular}{|c|c|c|c|c|c|c|}
\hline \multirow[t]{4}{*}{$\mathrm{s} 1$} & fst1 & mid1 & last1 & fst2 & mid2 & last2 \\
\hline & 0.85 & 0.95 & 1.0 & 0.75 & 0.9 & 1.3 \\
\hline & 0.95 & 1.0 & 0.85 & 0.9 & 1.3 & 0.75 \\
\hline & & & & \multicolumn{3}{|l|}{ dec } \\
\hline \multirow[t]{3}{*}{ s2 } & fst1 & last1 & idlep $^{p}$ & fst2 & mid2 & last2 \\
\hline & 0.0 & 0.95 & 1.0 & 0.75 & 0.9 & 1.3 \\
\hline & 0.95 & 0.0 & 0.85 & 0.9 & 1.3 & 0.75 \\
\hline
\end{tabular}

Fig. 5. Decrementing may result in an improper encoding. 
In a similar way to incrementing, there is also a rule corresponding to an instruction of the form $\left(s_{1}, c_{2}--, s_{2}\right)$.

Rotation To make it always possible to obtain a proper encoding after decrementing the value of a $c_{1}$ - or a $c_{2}$-encoding (see the decrementing rule above), we add a set of rotation rules. More precisely, for each state $s \in S$, the set $\mathcal{R}_{\mathcal{C}}$ contains the following two rules

$$
\begin{aligned}
& \operatorname{rot}_{2}^{s}:\left[\begin{array}{c}
s \\
\rightarrow \\
s
\end{array}\right]\left[\begin{array}{c}
\text { fst }_{2} \\
0<x_{1} \rightarrow \emptyset \\
\text { mid }_{2}
\end{array}\right]\left[\begin{array}{c}
\text { last }_{2} \\
x_{1}=1 \rightarrow\left\{x_{1}\right\} \\
\text { fst }_{2}
\end{array}\right]\left[\begin{array}{c}
\text { mid }_{2} \\
x_{2}=1 \rightarrow\left\{x_{2}\right\} \\
\text { last }_{2}
\end{array}\right] \\
& \left.\operatorname{rotz}_{2}^{s}:\left[\begin{array}{c}
s \\
\rightarrow \\
s
\end{array}\right]\left[\begin{array}{c}
f_{s t_{2}} \\
\left(0<x_{1}\right) \wedge\left(x_{2}=1\right) \\
\text { last }_{2}
\end{array}\right) \rightarrow\left\{x_{2}\right\}\right]\left[\begin{array}{c}
\text { last }_{2} \\
x_{1}=\underset{f}{1 \rightarrow}\left\{x_{1}\right\} \\
\text { fst }_{2}
\end{array}\right]
\end{aligned}
$$

Let us first explain the rule rots. The rule does not correspond to any instruction in $\mathcal{C}$; nor does it change the signature of the encoding. In simulating $\mathcal{C}$, we use the rotation rules in connection with decrementing. Recall that if $\theta=\left(s_{1}, c_{1}--, s_{2}\right)$ then applying a rule $d e c^{\theta}$ will not give a proper encoding in case the $c_{2}$-encoding has clocks with greater values than clock $x_{1}$ of the last process in the $c_{1}$-encoding (see Figure 5). The role of $\operatorname{rot}_{2}^{s}$ then is to decrement clock values of processes which are part of a $c_{2}$-encoding while preserving the signature of the whole encoding. More precisely, the rule rot $_{2}^{s}$ moves the process which is in state last $_{2}$ and makes it fi rst in the $\mathcal{C}_{2}$-encoding. This amounts to a rotation of the list corresponding to the $c_{2}$-encoding. The rotation can be repeated until suffi ciently many processes in the $\epsilon_{2}$-encoding have been moved. When there are no clocks in the $c_{2}$-encoding with greater clock values than clock $x_{1}$ of the last process in the $c_{1}$-encoding, the rotation stops and $\operatorname{dec}^{\theta}$ can now safely be applied.

We illustrate the role of $\operatorname{rot}_{2}^{s}$ through Figure 6. In a similar manner to Figure 5 we are interested in simulating a decrement instruction. However, instead of following the scenario of Figure 5, we now perform the following steps:

1. Wait for clock $x_{1}$ of the process in state last $_{2}$ to become equal to one (Figure 6(b)).

2. Apply the rule $\operatorname{rot}_{2}^{s_{1}}$. This results in an encoding (shown in Figure 6(c)) where clock $x_{1}$ of the process in state last $_{2}(0.6)$ is smaller than that of the process in state last $_{1}(0.7)$.

3. Wait until clock $x_{1}$ of the process in state last 1 becomes one (Figure 6(d)).

4. Apply the decrementing rule. Notice that the resulting encoding (shown in Figure $6(\mathrm{e}))$ has all clock values less than one.

After the last step, we can perform a timed transition and obtain a proper encoding.

Also if, before applying $\operatorname{dec}^{\theta}$, there is a clock in the $c_{2}$-encoding of the same value as clock $x_{1}$ of the process in state last $_{1}$, then we need to apply $\operatorname{rot}_{2}^{s_{2}}$ once more after decrementing (this scenario does not occur in Figure 6, but is considered in the correctness proof). 
(a)

\begin{tabular}{|c|c|c|c|c|c|c|}
\hline s1 & fst1 & mid1 & last1 & fst2 & $\operatorname{mid} 2$ & last2 \\
\hline & 0.2 & 0.3 & 0.35 & 0.1 & 0.25 & 0.65 \\
\hline & 0.3 & 0.35 & 0.2 & 0.25 & 0.65 & 0.1 \\
\hline
\end{tabular}

$\mathrm{T}=0.35$

(b)

\begin{tabular}{|l|l|l|l|l|l|l|}
\hline s1 & $\frac{\text { fst1 }}{0.55}$ & $\frac{\text { mid1 }}{0.65}$ & $\frac{\text { last1 }}{0.7}$ & $\frac{\text { fst2 }}{0.45}$ & $\frac{\text { mid2 }}{0.6}$ & $\frac{\text { last2 }}{1.0}$ \\
\hline 0.65 & $\frac{1.55}{0.6}$ & $\frac{0.45}{0.0}$ \\
\hline
\end{tabular}

$\operatorname{rot}_{2}^{\mathrm{sl}}$

(c)

\begin{tabular}{|c|c|c|c|c|c|c|}
\hline $\mathrm{s1}$ & fst1 & mid1 & last 1 & mid2 & last2 & fst2 \\
\hline & 0.55 & 0.65 & 0.7 & 0.45 & 0.6 & 0.0 \\
\hline & 0.65 & 0.7 & 0.55 & 0.6 & 0.0 & 0.45 \\
\hline
\end{tabular}

T $=0.3$

(d)

\begin{tabular}{|l|l|l|l|l|l|l|}
\hline s1 & $\frac{\text { fst1 }}{0.85}$ & $\frac{\text { mid1 }}{0.95}$ & $\frac{\text { last1 }}{1.0}$ \\
\hline$\frac{\text { mid2 }}{1.0}$ & $\frac{1 \text { last2 }}{0.85}$ & $\frac{\text { fst2 }}{0.9}$ & $\frac{0.9}{0.3}$ \\
\hline 0.3 & $\frac{0.75}{0.9}$ \\
\hline
\end{tabular}

dec

(e)

\begin{tabular}{|l|c|c|c|c|c|c|}
\hline s2 & fst1 \\
\hline$\frac{0.0}{\text { last1 }}$ & idle & $\frac{\mathrm{mid} 2}{0.95}$ & 1.0 & $\frac{0.75}{0.75}$ \\
\hline 0.95 & $\frac{1.0}{0.9}$ & $\frac{\text { fst2 }}{0.3}$ \\
\hline 0.0 & 0.85 & 0.9 & $\frac{0.3}{0.75}$ \\
\hline
\end{tabular}

Fig. 6. Decrementing preceded by rotation

Notice that we cannot apply the rotation rule in case the value of the $c_{2}$-encoding is zero. This is due to the fact that the rule requires at least one process in state mid $_{2}$. The rule $\operatorname{rotz}_{2}^{s}$ has the same role as $\operatorname{rot}_{2}^{s}$ with the difference that it can be applied when the value of the $c_{2}$-encoding is zero.

There are also similar rules $\operatorname{rot}_{1}^{s}$ and $\operatorname{rot}_{1}^{s}$ which are used to rotate a $c_{1}$-encoding and which are used in connection with rules of the form $\operatorname{dec}^{\theta}$ with $\theta=\left(s_{1}, c_{2}--, s_{2}\right)$.

Zero Testing For each instruction $\theta=\left(s_{1}, c_{1}=0\right.$ ?, $\left.s_{2}\right)$ in $\mathcal{C}$ there is a rule in $\mathcal{R}_{\mathcal{C}}$, namely

$$
t s t^{\theta}:\left[\begin{array}{c}
s_{1} \\
\rightarrow \\
s_{2}
\end{array}\right]\left[\begin{array}{c}
f_{s t_{1}} \\
\left(0<x_{1}\right) \wedge\left(x_{2}=1\right) \rightarrow\left\{x_{2}\right\} \\
\text { last }_{1}
\end{array}\right]\left[\begin{array}{c}
\text { last }_{1} \\
x_{1}=\underset{1 \rightarrow}{\left.1 \rightarrow x_{1}\right\}} \\
\text { fst }_{1}
\end{array}\right]
$$

The rule checks that the value of the encoding is zero by testing that there are no processes in state $m i d_{1}$. This is done by verifying that the process which is next last in the list is the same as the process which is fi rst in the list. We identify the next last process in a similar manner to the case with decrementing. More precisely, we wait until the value of clock $x_{1}$ of the last process is equal to one. At that moment, we check the 
process with clock $x_{2}$ equal to one, and check whether that process has a state equal to $f s t_{1}$. Notice that, clock $x_{2}$ of the fi rst process and clock $x_{1}$ of the last process are now both equal to one and the encoding is no more proper. In order to be able to obtain a proper encoding again, we reset both these clocks and interchange the states of the processes in states $f_{s t_{1}}$ and last $_{1}$ respectively.

Notice the similarity between the rules $t s t^{\theta}$ and $\operatorname{rot}_{1}^{s}$.

Some remarks about the rule $t s t^{\theta}$ :

- The rule $t s t^{\theta}$ is not enabled from an encoding $\gamma$ with $\operatorname{sig}(\gamma)=\left(s, m_{1}, m_{2}\right)$ and $m_{1} \neq 0$, since, in such an encoding, values of clocks $x_{1}$ of the process in state last $_{1}$ and $x_{2}$ of the process in state $f_{s t}$ are different.

- Sometimes the rule $t s t^{\theta}$ must be combined with the rotation rules according to the same scenarios explained for the decrementing rule.

In a similar way to the previous rules, there is also a rule corresponding to an instruction of the form $\theta=\left(s_{1}, c_{2}=0 ?, s_{2}\right)$.

Initialization The initial phase consists of the following two rules.

$$
\begin{aligned}
& \text { init }_{1}:\left[\begin{array}{c}
i d l e^{c} \\
\rightarrow \\
s_{\text {init }}^{\prime}
\end{array}\right]\left[\begin{array}{c}
i d l e^{p} \\
\text { true } \rightarrow\left\{x_{2}\right\} \\
{f s t_{1}}_{1}
\end{array}\right]\left[\begin{array}{c}
i d l e^{p} \\
\text { true } \rightarrow\left\{x_{2}\right\} \\
f_{s t_{2}}
\end{array}\right] \\
& {\left[\begin{array}{c}
i d l e^{p} \\
{\text { true } \rightarrow\left\{x_{1}\right\}}_{\text {last }_{1}}
\end{array}\right]\left[\begin{array}{c}
i d l e^{p} \\
{\text { true } \rightarrow\left\{x_{1}\right\}}_{\text {last }_{2}}
\end{array}\right]}
\end{aligned}
$$

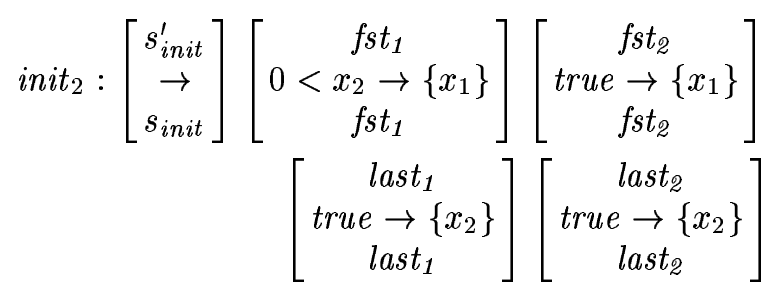

The role of the initialization rules is to bring $\mathcal{N}_{\mathcal{C}}$ from its initial confi guration (where the controller and all processes are idle) into a confi guration which is an encoding of the initial confi guration $\beta_{i n i t}$ of $\mathcal{C}$. The rule $i_{n i t}$ takes the controller into the temporary state $s_{i n i t}^{\prime}$. It also picks four processes such that two processes become the first and last processes in the $c_{1}$-encoding (with value zero) and the other two processes become the fi rst and last processes in the $c_{2}$-encoding (also with value zero). Clock $x_{2}$ of the first process and clock $x_{1}$ of the last process are reset. Rule init $_{2}$ changes the controller state to $s_{\text {init }}$ and completes the creation of the $c_{1}$-encoding and $c_{2}$-encoding. This is done by fi rst checking that some time has passed (through the guard $0<x_{2}$ ), and then resetting both clock $x_{1}$ of the process which is now in state $f s t_{1}\left(f s t_{2}\right)$, and clock $x_{2}$ of the process which is now in state last $_{1}$ (last $\left._{2}\right)$. 


\section{Correctness}

In this section we prove the correctness of the construction described in Section 4 and Section 5.

Let $\mathcal{C}=(S, \mathcal{I})$ be a counter machine and let $\mathcal{N}_{\mathcal{C}}=\left(Q_{\mathcal{C}}, \mathcal{R}_{\mathcal{C}}\right)$ a timed network derived from $\mathcal{C}$ as described in Section 4 and Section 5. Let $\leadsto$ and $\longrightarrow$ be the transition relations induced by $\mathcal{C}$ and $\mathcal{N}_{\mathcal{C}}$ respectively.

In this section, we show that if $s_{F}$ is a control state in $\mathcal{C}$ then the following holds.

Theorem 3. $\beta_{\text {init }} \stackrel{*}{\rightarrow} s_{F}$ iff $\gamma_{\text {init }} \stackrel{*}{\longrightarrow} s_{F}$ for some initial configuration $\gamma_{\text {init }}$ of $\mathcal{N}_{\mathcal{C}}$.

The proof of Theorem 3 is given in Subsections 6.1 and 6.2 each showing one direction of the equivalence.

In the proofs we need some defi nitions. Let $\mathcal{N}=(Q, \mathcal{R})$ be a timed network. We defi ne $\Longrightarrow_{r}$ to denote $\longrightarrow_{T} \circ \longrightarrow_{r} \circ \longrightarrow_{T}$, i.e. $\Longrightarrow_{r}$ corresponds to performing a discrete transition according to the rule $r$, preceded and followed by a timed transition. We defi ne $\Longrightarrow$ to be $\bigcup_{r \in \mathcal{R}} \Longrightarrow_{r}$. For a set $\mathrm{R} \subseteq \mathcal{R}$ of rules, we let $\gamma_{1} \Longrightarrow_{\mathrm{R}} \gamma_{2}$ denote that $\gamma_{1} \Longrightarrow_{r} \gamma_{2}$ for some $r \in \mathrm{R}$. We use $\stackrel{*}{\Longrightarrow}, \stackrel{*}{=} r$ and $\stackrel{*}{=}_{\mathrm{R}}$ to denote the reflexive transitive closure of the respective relations.

We introduce a new type of encodings to describe the effect of the rule $i n c_{1}^{\theta}$. For $m \geq 1$, a confi guration $\gamma=(I, q, \mathcal{Q}, X)$ is said to be a semi-q-encoding of value $m$ if there is an injection $h$ from $\{0, \ldots, m+1\}$ to $I$ such that the following conditions are satisfi ed

- $\mathcal{Q}$ is defi ned as in a $q$-encoding.

- $X_{2}(h(0))=X_{1}(h(1))<X_{2}(h(m+1))<X_{2}(h(1))$.

- $X_{1}(h(i))<X_{1}(h(i+1))$ for each $i: 2 \leq i \leq m$.

- $X_{2}(h(i))=X_{1}(h(i+1))$, for each $i: 1 \leq i \leq m$.

A graphical representation of a semi- $c_{1}$-encoding is shown in Figure 3(b).

In a similar manner to a $c_{1}$-encoding, we use $\operatorname{Val}_{1}(\gamma)$ to denote the value $m$ of a semi- $c_{1}$-encoding $\gamma$.

A confi guration $\gamma=(I, q, \mathcal{Q}, X)$ is said to be a Type 1 semi-encoding if it satisfi es the following two conditions:

$-q=t m p^{\theta}$ for some increment (of the form $\theta=\left(s_{1}, c_{1}++, s_{2}\right)$ or of the form $\left.\theta=\left(s_{1}, c_{2}++, s_{2}\right)\right)$.

$-\gamma$ is both a $c_{2}$-encoding and a semi- $c_{1}$-encoding.

In such a case, we defi ne $\operatorname{sig}(\gamma)$ of $\gamma$ to be the triple $\left(t m p^{\theta}, m_{1}, m_{2}\right)$, where $m_{1}=$ $\operatorname{Val}_{1}(\gamma)$ and $m_{2}=\operatorname{Val}_{2}(\gamma)$. Also, we defi ne $\operatorname{next}(\gamma)$ to be $\left(s_{2}, m_{1}, m_{2}\right)$. Intuitively, $\operatorname{next}(\gamma)$ is the signature of the confi guration which occurs next time we perform a discrete transition (by rule $i n c_{2}^{\theta}$ ) in our simulation.

The notion of a semi-encoding of Type 1 can be extended to a proper semi-encoding in the same manner as before, i.e. we require clocks of all processes which are not idle to have values strictly between zero and one. A (proper) semi-encoding of Type 2 is defi ned in a similar manner. 


\section{1 if-direction}

The if-direction follows immediately from the the following lemma.

Lemma 1. For any configuration $\gamma=(I, q, \mathcal{Q}, X)$ and initial configuration $\gamma_{\text {init }}$ in $\mathcal{N}_{\mathcal{C}}$, if $\gamma_{\text {init }} \stackrel{*}{\longrightarrow} \gamma$ then one of the following holds.

1. $q$ is not a member of $S$, (i.e. $q$ is either a temporary state or the state idle ${ }^{c}$ ).

2. $\gamma$ is an encoding such that $\beta_{\text {init }} \stackrel{*}{\sim} \operatorname{sig}(\gamma)$.

Proof. Suppose that $\gamma_{i n i t} \stackrel{*}{\longrightarrow} \gamma$. If $\gamma_{i n i t} \longrightarrow_{T} \gamma$ then the result follows immediately. Otherwise, $\gamma_{\text {init }} \stackrel{*}{\Longrightarrow} \gamma$, i.e., there is a sequence

$$
\gamma_{i n i t}=\gamma_{0} \Longrightarrow r_{0} \gamma_{1} \Longrightarrow \gamma_{r_{1}} \gamma_{2} \Longrightarrow r_{r_{2}} \cdots \Longrightarrow_{r_{n-1}} \gamma_{n}=\gamma
$$

Let $\gamma_{i}=\left(I, q_{i}, \mathcal{Q}_{i}, X_{i}\right)$ for $i: 0 \leq i \leq n$. We notice that $q_{0}=i d l e^{c}$. By defi nition of the rules, it must be the case that $r_{0}=i_{n i t} t_{1}$ and therefore $q_{1}=s_{\text {init }}^{\prime}$. In other words, both $\gamma_{0}$ and $\gamma_{1}$ satisfy the claim of the Lemma. Lemma 1 follows from the following property:

For each $2 \leq i \leq n$, it is the case that $\gamma_{i}$ is either

- an encoding with $\beta_{\text {init }} \stackrel{*}{\sim} \operatorname{sig}\left(\gamma_{i}\right)$; or

- a semi-encoding with $\beta_{\text {init }} \stackrel{*}{\sim} \operatorname{next}\left(\gamma_{i}\right)$.

This property is shown using an induction on $i$. For the base case we observe that, by defi nition of the rules, it follows that $\eta_{1}=$ init $_{2}$ and therefore $\operatorname{sig}\left(\gamma_{2}\right)=\beta_{\text {init }}$. For the induction step, we observe that, for each $i: 2 \leq i<n$, it follows from the rule defi nitions that one of the following cases is satisfi ed:

1. $r_{i}=i n c_{1}^{\theta}$ for some $\theta=\left(s_{1}, c_{1}++, s_{2}\right), \gamma_{i}$ is an encoding with $\operatorname{sig}\left(\gamma_{i}\right)=\left(s_{1}, m_{1}, m_{2}\right)$, and $\gamma_{i+1}$ is a Type 1 semi-encoding with $\operatorname{sig}\left(\gamma_{i+1}\right)=$ $\left(t m p^{\theta}, m_{1}+1, m_{2}\right)$.

2. $r_{i}=i n c_{2}^{\theta}$ for some $\theta=\left(s_{1}, c_{1}++, s_{2}\right), \gamma_{i}$ is a Type 1 semi-encoding with $\operatorname{sig}\left(\gamma_{i}\right)=\left(t m p^{\theta}, m_{1}, m_{2}\right)$, and $\gamma_{i+1}$ is an encoding with $\operatorname{sig}\left(\gamma_{i+1}\right)=$ $\left(s_{2}, m_{1}, m_{2}\right)$.

3. $r=\operatorname{dec}^{\theta}$ for some $\theta=\left(s_{1}, c_{1}--, s_{2}\right), \gamma_{i}$ is an encoding with $\operatorname{sig}\left(\gamma_{i}\right)=$ $\left(s_{1}, m_{1}, m_{2}\right), m_{1}>0$, and $\gamma_{i+1}$ is an encoding with $\operatorname{sig}\left(\gamma_{i+1}\right)=$ $\left(s_{2}, m_{1}-1, m_{2}\right)$.

4. $r=\operatorname{rot}_{1}^{s}$ for some $s \in S$ and $\operatorname{sig}\left(\gamma_{i}\right)=\operatorname{sig}\left(\gamma_{i+1}\right)$.

5. $r=\operatorname{rotz}_{1}^{s}$ for some $s \in S$ and $\operatorname{sig}\left(\gamma_{i}\right)=\operatorname{sig}\left(\gamma_{i+1}\right)$.

6. $r=t s t^{\theta}$ for some $\theta=\left(s_{1}, c_{1}=0\right.$ ?, $\left.s_{2}\right)$, with $\operatorname{sig}\left(\gamma_{i}\right)=\left(s_{1}, 0, m_{2}\right)$ and $\operatorname{sig}\left(\gamma_{i+1}\right)=\left(s_{2}, 0, m_{2}\right)$.

7. Similar cases corresponding to instructions which change counter $c_{2}$.

\section{2 only-if direction}

The only-if-direction follows from the following lemma. 
Lemma 2. If $\beta_{\text {init }} \stackrel{n}{\sim} s_{F}$ then $\gamma_{\text {init }} \stackrel{*}{\longrightarrow} s_{F}$, for each $n \geq 0$ and initial configuration $\gamma_{\text {init }}$ of $\mathcal{N}$ with $\left|\gamma_{\text {init }}\right| \geq n+4$.

The reason for the condition $\left|\gamma_{i n i t}\right| \geq n+4$ is that the sum of counter values never exceeds $n$ in the path from $\beta_{i n i t}$ to $s_{F}$. Furthermore, each $c_{1}$ - (or $c_{2}$ )-encoding uses $m+2$ processes for representing a counter value $m$. The lemma then states that the initial confi guration, from which we start the simulation of the path from $\beta_{i n i t}$ to $s_{F}$, should be suffi ciently large to incorporate all counter values which arise along that path.

Proof of Lemma 2 To show Lemma 2 we use some defi nitions.

Let $\gamma=(I, q, \mathcal{Q}, X)$ be a (semi-)encoding. Let $i \in I$ be the (unique) index such that $\mathcal{Q}(i)=$ last $_{1}$. We defi ne Latest $t_{1}(\gamma)=X_{1}(i)$. In other words, Latest ${ }_{1}(\gamma)$ is the highest among values of clocks belonging to processes which are part of the (semi$c_{1}$-encoding. We define Latest $t_{2}(\gamma)$ in a similar manner, and defi ne Latest $(\gamma)=$ $\max \left(\right.$ Latest $_{1}(\gamma)$, Latest $\left._{2}(\gamma)\right)$.

Let $\operatorname{Delay}_{1}(\gamma)$ be the size of the set $J \subseteq I$ such that $j \in J$ iff $\mathcal{Q}(j) \in$

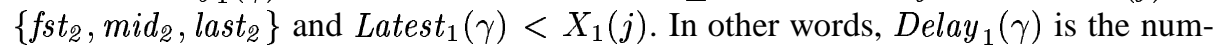
ber of processes which are part of the $c_{2}$-encoding and which have clocks with values higher than any clock of a process which is part of the $c_{1}$-encoding. We defi ne $\operatorname{Delay}_{2}(\gamma)$ in a similar manner. Notice that it may be the case that both $\operatorname{Delay}_{1}(\gamma)=0$ and $\operatorname{Delay}_{2}(\gamma)=0$ (if the maximum clock values are equal in the $c_{1}$ - and the $c_{2}$ encoding).

Lemma 2 follows immediately from the following lemma.

Lemma 3. For each $n \geq 0$ and initial configuration $\gamma_{\text {init }}$, if $\beta_{\text {init }} \stackrel{n}{\sim} \beta$ and $\left|\gamma_{i n i t}\right| \geq$ $n+4$, then there exists a proper encoding $\gamma$ such that $\gamma_{\text {init }} \stackrel{*}{\Longrightarrow} \gamma$ and $\operatorname{sig}(\gamma)=\beta$.

Proof. We prove this lemma by induction on $n$.

In the base case $(n=0)$, we have $\beta=\beta_{\text {init }}$ and $\left|\gamma_{i n i t}\right| \geq 4$. By the defi nition of init $_{1}$, this rule is enabled. Let $\gamma_{1}$ be such that $\gamma_{\text {init }} \longrightarrow_{\text {init }} \gamma_{1}$. Defi ne $\gamma_{2}=\gamma_{1}^{+t_{1}}$ with $0<t_{1}<1$. We have $\gamma_{1} \longrightarrow T_{T=t_{1}} \gamma_{2}$. Rule init $_{2}$ is now enabled. Let $\gamma_{3}$ be such that $\gamma_{2} \longrightarrow$ init $_{2} \gamma_{3}$. By defi nition of init $_{2}, \gamma_{3}$ is an encoding and $\operatorname{sig}\left(\gamma_{3}\right)=\beta_{\text {init }}$. Let $t_{2}$ be such that $0<t_{2}<1-\operatorname{Latest}\left(\gamma_{3}\right) . t_{2}$ exists by the defi nition of $t_{1}$, init $t_{1}$ and init $_{2}$. Let $\gamma_{4}=\gamma_{3}^{+t_{2}} \cdot \gamma_{4}$ is a proper encoding with $\operatorname{sig}\left(\gamma_{4}\right)=\beta_{i n i t}$. Notice that the transitions $\longrightarrow_{\text {init }_{1}}$ and $\longrightarrow$ init $_{2}$ are enabled only because $\left|\gamma_{i_{i n i t}}\right| \geq 4$.

For the induction step, assume that $\beta_{\text {init }} \stackrel{n+1}{\sim} \beta$ and $\left|\gamma_{i n i t}\right| \geq n+5$. We know that there is a $\beta_{1}$ with $\beta_{\text {init }} \stackrel{n}{\sim} \beta_{1} \leadsto \beta$. By the induction hypothesis, it follows that there is a proper encoding $\gamma_{1}$ such that $\operatorname{sig}\left(\gamma_{1}\right)=\beta_{1}$ and $\gamma_{i n i t} \stackrel{*}{\Rightarrow} \gamma_{1}$. We need to show that there is a proper encoding $\gamma$ with $\operatorname{sig}(\gamma)=\beta$ and $\gamma_{1} \stackrel{*}{\Rightarrow} \gamma$. This follows from the following lemma.

Lemma 4. Let $\beta_{1}$ and $\beta_{2}$ be configurations of $\mathcal{C}$, where $\beta_{1} \leadsto \beta_{2}$ and $\beta_{2}$ is of the form $\left(s, m_{1}, m_{2}\right)$. Let $\gamma_{1}$ be a proper encoding such that sig $\left(\gamma_{1}\right)=\beta_{1}$ and $\left|\gamma_{1}\right| \geq$ $m_{1}+m_{2}+4$. There is a proper encoding $\gamma_{2}$ such that $\operatorname{sig}\left(\gamma_{2}\right)=\beta_{2}$ and $\gamma_{1} \stackrel{*}{\Longrightarrow} \gamma_{2}$.

The proof of Lemma 4 follows from Lemma 5, Lemma 6, Lemma 9, and Lemma 10: 
- Lemma 5 and Lemma 6 state that an increment can be simulated by an application of the rule $i n c_{1}^{\theta}$ followed by an application of the rule $i n c_{2}^{\theta}$.

- Lemma 9 states that a decrement can be simulated by the rule $d e c^{\theta}$ possibly preceded and followed by a number of rotations. This lemma follows from Lemma 7 and Lemma 8.

- Lemma 10 deals with zero testing and is similar to Lemma 9.

The condition $\left|\gamma_{1}\right| \geq m_{1}+m_{2}+4$ in the claim of Lemma 4 is relevant only in Lemma 5, since this is the only case where the value of a counter is increased.

Lemma 5. Consider an instruction $\theta=\left(s_{1}, c_{1}++, s_{2}\right)$. Let $\gamma_{1}$ be a proper encoding with $\operatorname{sig}\left(\gamma_{1}\right)=\left(s_{1}, m_{1}, m_{2}\right)$ and $\left|\gamma_{1}\right| \geq m_{1}+m_{2}+5$. There is a proper semi-encoding $\gamma_{2}$ of Type 1 such that $\operatorname{sig}\left(\gamma_{2}\right)=\left(t m p^{\theta}, m_{1}+1, m_{2}\right)$ and $\gamma_{1} \Longrightarrow_{i n c_{1}^{\theta}} \gamma_{2}$.

A similar result holds in case $\theta$ is of the form $\left(s_{1}, c_{2}++, s_{2}\right)$.

Proof. Since $\left|\gamma_{1}\right| \geq m_{1}+m_{2}+5$, there is at least one process in $\gamma_{1}$ whose state is $i d l e^{p}$ (we need $m_{1}+2$ processes for the $c_{1}$-encoding and $m_{2}+2$ processes for the $c_{2}$-encoding, which means that we have at least one process left to be in state $i d l e^{p}$ ). This together with the fact that $\gamma_{1}$ is a proper encoding implies that $i n c_{1}^{\theta}$ is enabled from $\gamma_{1}$, i.e., there is confi guration $\gamma_{3}$ with $\gamma_{1} \longrightarrow_{i n c_{1}^{\theta}} \gamma_{3}$. Defi ne $\gamma_{2}=\gamma_{3}^{+t}$ where $0<t<1-\operatorname{Latest}\left(\gamma_{3}\right)$. Such a $t$ exists by defi nition of $i n \epsilon_{1}^{\theta}$ and since $\gamma_{1}$ is a proper encoding. By the defi nitions it follows that $\gamma_{2}$ is a proper semi-encoding of Type 1 with $\operatorname{sig}\left(\gamma_{2}\right)=\left(t m p^{\theta}, m_{1}+1, m_{2}\right)$ and $\gamma_{1} \longrightarrow_{i n c_{1}^{\theta}} \gamma_{3} \longrightarrow_{T=t} \gamma_{2}$.

Lemma 6. Consider an instruction $\theta=\left(s_{1}, c_{1}++, s_{2}\right)$. Let $\gamma_{1}$ be a proper semiencoding of Type 1 with sig $\left(\gamma_{1}\right)=\left(t m p^{\theta}, m_{1}, m_{2}\right)$. There is a proper encoding $\gamma_{2}$ such that $\operatorname{sig}\left(\gamma_{2}\right)=\left(s_{2}, m_{1}, m_{2}\right)$ and $\gamma_{1} \Longrightarrow{ }_{\text {inc }}^{\theta} \gamma_{2}$.

A similar result holds in case $\theta$ is of the form $\left(s_{1}, c_{2}++, s_{2}\right)$.

Proof. Since $\gamma_{1}$ is a proper semi-encoding of Type 1, it follows that $i n c_{2}^{\theta}$ is enabled from $\gamma_{1}$, i.e., there is a confi guration $\gamma_{3}$ with $\gamma_{1} \longrightarrow_{i n c_{2}^{\theta}} \gamma_{3}$. Defi ne $\gamma_{2}=\gamma_{3}^{+t}$ where $0<t<1-\operatorname{Latest}\left(\gamma_{3}\right)$. Such a $t$ exists by defi nition of $i n c_{2}^{\theta}$ and since $\gamma_{1}$ is proper semi-encoding. By the defi nitions it follows that $\gamma_{2}$ is a proper encoding with $\operatorname{sig}\left(\gamma_{2}\right)=$ $\left(s_{2}, m_{1}, m_{2}\right)$ and $\gamma_{1} \longrightarrow_{i n c_{2}^{\theta}} \gamma_{3} \longrightarrow_{T=t} \gamma_{2}$.

Lemma 7. Let $\gamma_{1}$ be a proper encoding with Delay ${ }_{1}\left(\gamma_{1}\right)>0$ and $\operatorname{sig}\left(\gamma_{1}\right)=$ $\left(s, m_{1}, m_{2}\right)$. There is a proper encoding $\gamma_{2}$ such that Delay ${ }_{1}\left(\gamma_{2}\right)=\operatorname{Delay}_{1}\left(\gamma_{1}\right)-1$, $\operatorname{sig}\left(\gamma_{1}\right)=\operatorname{sig}\left(\gamma_{2}\right)$, and either $\gamma_{1} \Longrightarrow_{\text {rot }_{2}^{s}} \gamma_{2}$ or $\gamma_{1} \Longrightarrow_{\text {rotz }_{2}^{s}} \gamma_{2}$.

A similar result holds in case Delay ${ }_{2}\left(\gamma_{1}\right)>0$.

Proof. We distinguish between two cases, namely when $m_{2}>0$ and when $m_{2}=0$.

First, we assume that $m_{2}>0$. Defi ne $\gamma_{3}=\gamma_{1}^{+t_{1}}$ where $t_{1}=1-$ Latest $_{2}\left(\gamma_{1}\right)$. Such a $t_{1}$ exists since $\gamma_{1}$ is a proper encoding. From the defi nition of $t_{1}$ and the fact that $m_{2}>$ 0 it follows that rot $_{2}^{s}$ is enabled from $\gamma_{3}$, i.e., there is a $\gamma_{4}$ with $\gamma_{3} \longrightarrow_{\text {rot }_{2}^{s}} \gamma_{4}$. Defi ne $\gamma_{2}=\gamma_{4}^{+t_{2}}$ where $0<t_{2}<1-\operatorname{Latest}\left(\gamma_{4}\right)$. Existence of $t_{2}$ follows from the manner in which $t_{1}$ is chosen, defi nition of the rule rot $t_{2}^{s}$, and since $\gamma_{1}$ is a proper encoding. By the defi nitions it follows that $\gamma_{2}$ is a proper encoding with $\operatorname{Delay}_{1}\left(\gamma_{2}\right)=\operatorname{Delay}_{1}\left(\gamma_{1}\right)-1$, and $\operatorname{sig}\left(\gamma_{2}\right)=\operatorname{sig}\left(\gamma_{1}\right)$. 
The case when $m_{2}=0$ is similar. Here we replace the rule $\operatorname{rot}_{2}^{s_{2}}$ by the rule $\operatorname{rot}_{2}^{s_{2}}$, and obtain $\gamma_{1} \longrightarrow_{T=t_{1}} \gamma_{3} \longrightarrow_{r o t z}{ }_{2}^{s} \gamma_{4} \longrightarrow T=t_{2} \gamma_{2}$.

Lemma 8. Consider an instruction $\theta=\left(s_{1}, c_{1}--, s_{2}\right)$. Let $\gamma_{1}$ be a proper encoding with $\operatorname{sig}\left(\gamma_{1}\right)=\left(s_{1}, m_{1}, m_{2}\right)$ and $m_{1}>0$. If Delay $y_{1}\left(\gamma_{1}\right)=0$ then there is a proper encoding $\gamma_{2}$ such that sig $\left(\gamma_{2}\right)=\left(s_{2}, m_{1}-1, m_{2}\right)$ and one of the following holds.

1. If Latest ${ }_{1}\left(\gamma_{1}\right)>$ Latest $_{2}\left(\gamma_{1}\right)$ then $\gamma_{1} \Longrightarrow{ }_{d e c^{\theta}} \gamma_{2}$.

2. If Latest ${ }_{1}\left(\gamma_{1}\right)=$ Latest $_{2}\left(\gamma_{1}\right)$ and $m_{2}>0$ then $\gamma_{1} \Longrightarrow_{\text {dec }^{\theta}} \circ \Longrightarrow_{\text {rot }_{2}^{s_{2}}} \gamma_{2}$.

3. If Latest ${ }_{1}\left(\gamma_{1}\right)=$ Latest $_{2}\left(\gamma_{1}\right)$ and $m_{2}=0$ then $\gamma_{1} \Longrightarrow_{\text {dec }^{\theta}} \circ \Longrightarrow_{\text {rotz }_{2}^{s_{2}}} \gamma_{2}$.

A similar result holds in case $\theta$ is of the form $\left(s_{1}, c_{2}--, s_{2}\right)$.

Proof. Defi ne $\gamma_{3}=\gamma_{1}^{+t_{1}}$ where $t_{1}=1-$ Latest $_{1}\left(\gamma_{1}\right)$. Such a $t_{1}$ exists since $\gamma_{1}$ is a proper encoding. From the defi nition of $t_{1}$ and the fact that $m_{1}>0$ it follows that $d e c^{\theta}$ is enabled from $\gamma_{3}$, i.e., there is a $\gamma_{4}$ with $\gamma_{3} \longrightarrow{ }_{d e c^{\theta}} \gamma_{4}$. Now there are three cases depending on the values of Latest $_{1}\left(\gamma_{1}\right)$ and Latest ${ }_{2}\left(\gamma_{1}\right)$ as follows:

1. If Latest ${ }_{1}\left(\gamma_{1}\right)>$ Latest $_{2}\left(\gamma_{1}\right)$ then define $\gamma_{2}=\gamma_{4}^{+t_{2}}$ where $0<t_{2}<1-$ Latest $\left(\gamma_{4}\right)$. Existence of $t_{2}$ follows from the manner in which $t_{1}$ is chosen, definition of the rule $d e c^{\theta}$, and since $\gamma_{1}$ is a proper encoding. By the defi nitions it follows that $\gamma_{2}$ is a proper encoding with $\operatorname{sig}\left(\gamma_{2}\right)=\left(s_{2}, m_{1}-1, m_{2}\right)$, and $\gamma_{1} \longrightarrow_{T=t_{1}} \gamma_{3} \longrightarrow_{\text {dec }^{\theta}} \gamma_{4} \longrightarrow_{T=t_{2}} \gamma_{2}$.

2. If Latest $1\left(\gamma_{1}\right)=$ Latest $_{2}\left(\gamma_{1}\right)$ and $m_{2}>0$. It follows that clock $x_{1}$ of the last process in the $c_{2}$-encoding has value 1 in $\gamma_{4}$. Therefore, the rule $\operatorname{rot}_{2}^{s_{2}}$ is enabled from $\gamma_{4}$, i.e., there is a $\gamma_{5}$ with $\gamma_{4} \longrightarrow{ }_{r o t} t_{2}^{s_{2}} \gamma_{5}$. Defi ne $\gamma_{2}=\gamma_{5}^{+t_{2}}$ where $0<t_{2}<$ $1-$ Latest $_{1}\left(\gamma_{5}\right)$. Existence of $t_{2}$ follows from the manner in which $t_{1}$ is chosen, defi nitions of the rules $\operatorname{dec}^{\theta}$ and $\operatorname{rot}_{2}^{s_{2}}$, and since $\gamma_{1}$ is a proper encoding. By the defi nitions it follows that $\gamma_{2}$ is a proper encoding with $\operatorname{sig}\left(\gamma_{2}\right)=\left(s_{2}, m_{1}-1, m_{2}\right)$, and $\gamma_{1} \longrightarrow_{T=t_{1}} \gamma_{3} \longrightarrow_{d e c^{\theta}} \gamma_{4} \longrightarrow_{r o t}{ }_{2}^{s_{2}} \gamma_{5} \longrightarrow_{T=t_{2}} \gamma_{2}$.

3. If Latest ${ }_{1}\left(\gamma_{1}\right)=$ Latest $_{2}\left(\gamma_{1}\right)$, but $m_{2}=0$. The proof is similar to the previous case. Here, we use the rule rotz $z_{2}^{s_{2}}$ instead of the rule $\operatorname{rot}_{2}^{s_{2}}$ and obtain $\gamma_{1} \longrightarrow_{T=t_{1}}$ $\gamma_{3} \longrightarrow \operatorname{dec}^{\theta} \gamma_{4} \longrightarrow_{r o t z} z_{2}^{s_{2}} \gamma_{5} \longrightarrow{ }_{T=t_{2}} \gamma_{2}$.

From Lemma 7 and Lemma 8 we get the following.

Lemma 9. Consider an instruction $\theta=\left(s_{1}, c_{1}--, s_{2}\right)$. Let $\gamma_{1}$ be a proper encoding with $\operatorname{sig}\left(\gamma_{1}\right)=\left(s_{1}, m_{1}, m_{2}\right)$ and $m_{1}>0$. There is a proper encoding $\gamma_{2}$ such that $\operatorname{sig}\left(\gamma_{2}\right)=\left(s_{2}, m_{1}-1, m_{2}\right)$ and

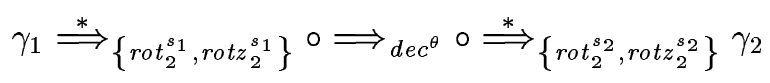

A similar result holds in case $\theta$ is of the form $\left(s_{1}, c_{2}--, s_{2}\right)$.

The proof of the following lemma is similar to the proof of Lemma 9.

Lemma 10. Consider an instruction $\theta=\left(s_{1}, c_{1}=0\right.$ ?, $\left.s_{2}\right)$. Let $\gamma_{1}$ be a proper encoding with $\operatorname{sig}\left(\gamma_{1}\right)=\left(s_{1}, 0, m_{2}\right)$. Then there is a proper encoding $\gamma_{2}$ such that $\operatorname{sig}\left(\gamma_{2}\right)=\left(s_{2}, 0, m_{2}\right)$ and

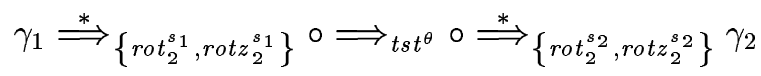

A similar result holds in case $\theta$ is of the form $\left(s_{1}, c_{2}=0 ?, s_{2}\right)$. 


\section{Discrete Timed Networks}

In this section, we show decidability of the controller state reachability problem for Discrete Timed Networks (DTNs): timed networks in which the clocks assume values from the set of natural numbers. The idea of the proof is to defi ne an ordering on confi gurations of the DTN. The ordering amounts to counter abstraction: for each confi guration we count the number of processes which are in a given state and whose clocks are equal to some given values.

Discrete Timed Networks (DTN) The syntax of a DTN is the same as that of a TN (see Section 2). A confi guration is also of the same form as in a TN. The behaviour of a DTN differs from that of a TN in two aspects, namely

- In a confi guration $(I, q, \mathcal{Q}, X)$, the type of $X$ is $\{1, \ldots, K\} \rightarrow I \rightarrow \mathbb{N}$, i.e., clocks have values which are natural numbers rather than reals.

- Timed transitions take only discrete steps, i.e., $\gamma_{1} \longrightarrow_{T=t} \gamma_{2}$ if $\gamma_{2}=\gamma_{1}^{+t}$ where $t \in \mathbb{N}$. Discrete transitions are defi ned in a similar manner to TN.

Thus, the transition relation $\longrightarrow$ defi ned in Section 2 is adapted to DTN as described above.

Ordering: We defi ne an ordering $\preceq$ on the set of confi gurations $\Gamma$ of a DTN $\mathcal{N}$ with $K$ clocks as follows. To defi ne $\preceq$, we fi rst introduce a function

$$
\#: Q^{\text {proc }} \times\{0, \ldots, \max +1\}^{K} \rightarrow \Gamma \rightarrow \mathbb{N}
$$

where $\max$ is the maximum natural number which occurs in the defi nitions of the rules in the DTN. Given $p \in Q^{\text {proc }}, m_{1}, \ldots, m_{K} \in\{0, \ldots, \max +1\}$, and $\gamma=(I, q, \mathcal{Q}, X)$ we defi ne \# $\left(p, m_{1}, \ldots, m_{K}\right)(\gamma)$ to be the size of the set $I_{1} \subseteq I$ such that for each index $i \in I_{1}, \mathcal{Q}(i)=p$ and for each $k: 1 \leq k \leq K$, one of the following conditions holds

- $X(k)(i)=m_{k}$ and $m_{k} \leq \max$.

- $X(k)(i)>m_{k}$ and $m_{k}=\max +1$.

In other words, \# $\left(p, m_{1}, \ldots, m_{K}\right)(\gamma)$ counts the number of processes in $\gamma$ whose states are $p$ and whose clock values are given by $m_{1}, \ldots, m_{K}$ respectively (we identify clock values larger than max). For $\gamma=(I, q, \mathcal{Q}, X)$ and $\gamma^{\prime}=\left(I^{\prime}, q^{\prime}, \mathcal{Q}^{\prime}, X^{\prime}\right) \in \Gamma$, we use $\gamma \preceq \gamma^{\prime}$ to denote that the following two conditions hold:

$-q=q^{\prime}$

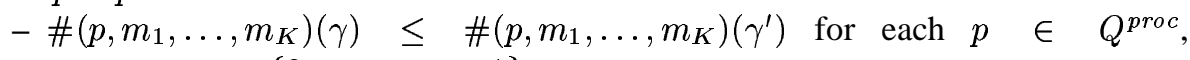
$m_{1}, \ldots, m_{K} \in\{0, \ldots, \max +1\}$.

Thus, the ordering $\preceq$ is trivially computable.

Well-quasi-ordering: An ordering $\preceq$ on the set of confi gurations is a well-quasiordering if for each infi nite sequence $\gamma_{0}, \gamma_{1}, \gamma_{2}, \ldots$ of confi gurations, there are $i$ and $j$ with $i<j$ and $\gamma_{i} \preceq \gamma_{j}$. Well quasi-ordering of $\preceq$ follows from Dickson's Lemma [Dic13]: according to the ordering, we can represent a confi guration by a vector of natural numbers, where each entry of the vector is indexed by a tuple of the form $\left(p, m_{1}, \ldots, m_{K}\right)$. 
Lemma 11. The ordering $\preceq$ on the set of configurations of a DTN is a well-quasiordering.

Lemma 12. The transition relation $\longrightarrow$ over the set of configurations of a DTN is monotonic wrt $\preceq$.

i.e, given confi gurations $\mathfrak{x}, \gamma_{2}, \gamma_{3}$ such that $\gamma_{1} \longrightarrow \gamma_{2}$ and $\gamma_{1} \preceq \gamma_{3}$, there is a confi guration $\gamma_{4}$ such that $\gamma_{3} \longrightarrow \gamma_{4}$ and $\gamma_{2} \preceq \gamma_{4}$.

Proof. To show that $\longrightarrow$ is monotonic with respect to $\preceq$, we show that both $\longrightarrow{ }_{r}$ and $\longrightarrow_{T}$ are monotonic with respect to $\preceq$.

Monotonicity of $\longrightarrow_{r}$ is straightforward. So we only show the monotonicity of $\longrightarrow_{T}$. Given $\gamma_{1}, \gamma_{2}$ such that $\gamma_{1} \preceq \gamma_{2}$, we show that $\gamma_{1}^{+t} \preceq \gamma_{2}^{+t}$.

- Given $p \in Q^{\text {proc }}, m_{1}^{\prime}, \ldots, m_{K}^{\prime} \in\{1, \ldots, \max +1\}$, the defi nition of $\longrightarrow T$ and the fact that the clocks with values larger than $\max$ are identifi ed, it follows that $\#\left(p, m_{1}^{\prime}, \ldots, m_{K}^{\prime}\right)\left(\gamma^{+t}\right)=\quad \Sigma_{m_{1}, \ldots, m_{K}} \#\left(p, m_{1}, \ldots, m_{K}\right)(\gamma)$ such that one of the following holds for each $k: 1 \leq k \leq K$.

- if $m_{k}^{\prime} \leq \max$, then $m_{k}=m_{k}^{\prime}-t$.

- if $m_{k}^{\prime}=\max +1$, then $\max +1-t \leq m_{k} \leq \max +1$.

- Given $\gamma_{1} \preceq \gamma_{2}$, for each $p \in Q^{\text {proc }}, m_{1}, \ldots, m_{K} \in\{0, \ldots, \max +1\}$, we have

$$
\#\left(p, m_{1}, \ldots, m_{K}\right)\left(\gamma_{1}\right) \leq \#\left(p, m_{1}, \ldots, m_{K}\right)\left(\gamma_{2}\right)
$$

- Above two conditions imply that for each $p \in Q^{\text {proc }}, m_{1}^{\prime}, \ldots, m_{K}^{\prime} \in$ $\{0, \ldots, \max +1\}$, we have

$$
\#\left(p, m_{1}^{\prime}, \ldots, m_{K}^{\prime}\right)\left(\gamma_{1}^{+t}\right) \leq \#\left(p, m_{1}^{\prime}, \ldots, m_{K}^{\prime}\right)\left(\gamma_{2}^{+t}\right)
$$

i.e, $\gamma_{1}^{+t} \preceq \gamma_{2}^{+t}$.

The Problem DTN $(K)$-Reach is defi ned in the same manner as TN $(K)$-Reach except that the timed network $\mathcal{N}$ in the defi nition of the problem is now given a discrete interpretation as described above.

In this section we show

Theorem 4. $D T N(K)$-Reach is decidable for each $K \in \mathbb{N}$.

To prove Theorem 4, we rely on the theory introduced in [AČJYK00].

Monotonic Transition Systems (MTS) A monotonic transition system (MTS) is a tuple $(\Gamma$, Init $, \preceq, \hookrightarrow, U)$, where

- $\Gamma$ is a (potentially infi nite) set of configurations.

- Init $\subseteq \Gamma$ is a set of initial confi gurations.

- $\preceq$ is a computable ordering on $\Gamma$, i.e., for each $\gamma_{1}, \gamma_{2} \in \Gamma$, we can check whether $\gamma_{1} \preceq \gamma_{2}$. Furthermore, $\preceq$ is a well quasi-ordering

- $\hookrightarrow$ is a binary transition relation on $\Gamma$. Furthermore, $\hookrightarrow$ is monotonic with respect to $\preceq$, 
- $U$ is defi ned as the upward closure $\Gamma_{1} \uparrow$ of a fi nite set $\Gamma_{1} \subseteq \Gamma$, where $\Gamma_{1} \uparrow=$ $\left\{\gamma^{\prime} \in \Gamma \mid \exists \gamma \in \Gamma_{1} . \gamma \preceq \gamma^{\prime}\right\}$.

We use $\stackrel{*}{\hookrightarrow}$ to denote the the reflexive transitive closure of $\hookrightarrow$. For sets $\Gamma_{1}, \Gamma_{2} \subseteq \Gamma$, we say that $\Gamma_{2}$ is reachable from $\Gamma_{1}$ if there are $\gamma_{1} \in \Gamma_{1}$ and $\gamma_{2} \in \Gamma_{2}$ such that $\gamma_{1} \stackrel{*}{\hookrightarrow} \gamma_{2}$.

The reachability problem for MTS (MTS-Reach) is defi ned as follows:

Instance An MTS $(\Gamma$, Init, $\preceq, \hookrightarrow, U)$.

Question Is $U$ reachable from Init?

In [AČJYK00] we give suffi cient conditions for decidability of MTS-Reach as follows. For $\Gamma_{1} \subseteq \Gamma$, we defin ne $\operatorname{Pre}\left(\Gamma_{1}\right)$ to be the set $\left\{\gamma \mid \exists \gamma_{1} \in \Gamma_{1} \cdot \gamma \hookrightarrow \gamma_{1}\right\}$. For $\Gamma_{1} \subseteq \Gamma$, we say that $M \subseteq \Gamma_{1}$ is minor set of $\Gamma_{1}$ if

- for each $\gamma_{1} \in \Gamma_{1}$ there is $\gamma_{2} \in M$ such that $\gamma_{2} \preceq \gamma_{1}$.

- If $\gamma_{1}, \gamma_{2} \in M$ and $\gamma_{1} \preceq \gamma_{2}$ then $\gamma_{1}=\gamma_{2}$.

Since $\preceq$ is a wqo, it follows that each minor set is fi nite. However, for the same set, there may be several minor sets. We use $\min$ to denote a function which, given $\Gamma_{1} \subseteq \Gamma$, returns a minor set of $\Gamma_{1}$. We use minpre $(\gamma)$ to denote the set $\min (\operatorname{Pre}(\{\gamma\} \uparrow))$.

In [AČJYK00] we show that the following conditions are suffi cient for decidability of MTS-Reach.

Theorem 5. MTS-Reach is decidable if for each $\gamma \in \Gamma$

- we can check whether $\gamma \in$ Init.

- the set minpre $(\gamma)$ is finite and computable.

\section{From DTN to MTS}

A DTN $\mathcal{N}=(Q, \mathcal{R})$ with $K$ clocks, together with a controller state $q_{F}$ induces an $\operatorname{MTS}(\Gamma$, Init $, \preceq, \hookrightarrow, U)$ as follows

- $\Gamma$ is the set of confi gurations of $\mathcal{N}$.

- Init is the set of initial confi gurations of $\mathcal{N}$.

- By Lemma $11, \preceq$ is computable and it is a well-quasi-ordering.

- $\hookrightarrow$ is the relation $\longrightarrow$ defi ned in Section 2 adapted to DTN as described above. Furthermore, $\longrightarrow$ is monotonic wrt $\preceq$ by Lemma 12 .

- $U=\left\{\left(\emptyset, q_{F}, \mathcal{Q}, X\right)\right\} \uparrow$, i.e., $U$ is the set of all confi gurations with controller state $q_{F}$.

This means that a DTN indeed induces an MTS.

Notice that it is trivial to check whether a given confi guration is initial. The following lemma states that the induced transition system also satisfi es the second suffi cient condition for decidability (see Theorem 5).

Lemma 13. Consider the MTS induced by a DTN. Then, for each configuration $\gamma$ we can compute minpre $(\gamma)$ as a finite set of configurations. 
Proof. Given a confi guration $\gamma$, we compute minpre $\left(\gamma^{\prime}\right)$ as follows. Since, $\longrightarrow=\longrightarrow T$ $\cup \bigcup_{r \in \mathcal{R}} \longrightarrow{ }_{r}$, we compute minpre $\left(\gamma^{\prime}\right)=$ minpre $_{T}\left(\gamma^{\prime}\right) \cup \bigcup_{r \in \mathcal{R}}$ minpre $_{r}\left(\gamma^{\prime}\right)$ where minpre $_{T}\left(\gamma^{\prime}\right)$ characterizes the set of confi gurations from which we reach $\gamma \uparrow$ through passage of time and minpre $_{r}\left(\gamma^{\prime}\right)$ does the same through execution of a rule $r$.

For a confi guration $\gamma=(I, q, \mathcal{Q}, X)$, we defi ne $\operatorname{minc}(\gamma)$ to be the minimum value in the set $\left\{X_{k}(i) \mid 1 \leq k \leq K \wedge i \in I\right\}$, i.e, $\operatorname{minc}(\gamma)$ denotes the minimum clock value which occurs in $\gamma$. We compute minpre ${ }_{T}(\gamma)$ as follows.

$$
\operatorname{minpre}_{T}(\gamma)=\left\{\gamma^{-t} \mid 0 \leq t \leq \operatorname{minc}(\gamma)\right\}
$$

where $\gamma^{-t}$ is defi ned in a manner similar to the defi nition of $\gamma^{t}$.

Next, we show how to compute minpre $r_{r}$. Given a configuration $\gamma=$ $\left\langle I^{\prime}, q_{0}^{\prime}, \mathcal{Q}^{\prime}, X^{\prime}\right\rangle$ and a rule $r \in \mathcal{R}$ of the form

$$
\left[\begin{array}{c}
q_{0} \\
\overrightarrow{q_{0}^{\prime}}
\end{array}\right]\left[\begin{array}{c}
q_{1} \\
g_{1} \underset{\rightarrow}{\rightarrow} R_{1} \\
q_{1}^{\prime}
\end{array}\right] \quad \cdots \quad\left[\begin{array}{c}
q_{n} \\
g_{n} \underset{\rightarrow}{\rightarrow} R_{n} \\
q_{n}^{\prime}
\end{array}\right]
$$

we compute a fi nite set of confi gurations $\Gamma$ such that $\operatorname{minpre}\left(\gamma^{\prime}\right)=\min (\Gamma)$. We defi ne $\Gamma$ to be the set of all confi gurations of the form $\langle I, \mathbb{q}, \mathcal{Q}, X\rangle$ such that there are three pairwise disjoint sets changing, unchanged and guarding of indices and the following holds.

- $I^{\prime}=$ changing $\cup$ unchanged,

- I = changing $\cup$ unchanged $\cup$ guarding, and

- there is a bijection $h:$ changing $\cup$ guarding $\rightarrow\{1, \ldots, n\}$, which satisfi es the following conditions.

1. $\mathcal{Q}(j)=q_{h(j)}$, and the guard $g_{h(j)}\left(X_{1}(j), \ldots, X_{K}(j)\right)$ holds. Furthermore, $X(k)(j) \in\{0, \ldots, \max +1\}$, for each $j \in$ (changing $\cup$ guarding $)$ and for each $k: 1 \leq k \leq K$.

2. $\mathcal{Q}^{\prime}(j)=q_{h(j)}^{\prime}$ for $j \in$ changing.

3. $\mathcal{Q}^{\prime}(j)=\mathcal{Q}(j)$ for $j \in$ unchanged.

4. For each $k: 1 \leq k \leq K, X_{k}^{\prime}(j)=0$ if $j \in$ changing and $x_{k} \in R_{h(j)}$.

5. For each $k: 1 \leq k \leq K$ and for each $j$ such that either $j \in$ changing and $x_{k} \notin R_{h(j)}$, or $j \in$ unchanged, $X_{k}(j)=\min \left(\max +1, X_{k}^{\prime}(j)\right)$.

Observe that

- The set guarding of indices with size $m$ can correspond to infi nitely many sets of the same size. We equate all of them modulo renaming. Therefore, the sets changing, unchanged, guarding are fi nite and effectively constructible.

- The conditions on the controller states are implicitly included by our notation, which requires the controller states of $\gamma$ and $\gamma^{\prime}$ to be the controller states of $r$.

- The correctness of the above algorithm can be shown in a similar manner to the proofs in [AJ03].

This, together with Theorem 5, proves Theorem 4 . 


\section{Conclusions, Discussion, and Future Work}

We have shown undecidability of controller state reachability for multi-clock timed networks. We have also shown decidability of the problem when clocks are interpreted over a discrete time domain.

In this paper, we assume a lazy behaviour for TNs. This means that we may choose to let time pass instead of performing discrete transitions, even if that makes these transitions disabled, due to some of the clocks becoming "too old". In fact, we can use the techniques in [JLL77] to show that, in the case of urgent behaviour, the controller state reachability is undecidable even for single-clock TNs. Also, in this paper we only consider safety properties. Liveness properties have been shown to be undecidable for single-clock TNs in [AJ03].

The ordering we provide for proving decidability of DTN corresponds to an abstraction of confi gurations where we count the number of processes which are in a certain state and which have certain clock values. In a similar manner to [GS92] we can view this abstraction as a "Petri net"-like model where each place corresponds to one combination of process states and clock values. In contrast to [GS92], the transitions in the abstract model do not correspond to those of a Petri net. The main difference is that a timed transition simultaneously moves all tokens from each place, corresponding to a certain clock value, to the place corresponding to the next clock value. Comparing to the model of Transfer Nets [FRSB02], a timed transition here corresponds to "parallel transfers", i.e. a set of transfers which are performed simultaneously. An alternative way to prove our decidability result would be to simulate a DTN by a transfer net. One ingredient in such a simulation is to simulate parallel transfers by sequences of transfer operations.

There are several classes of protocols which can be modelled as multi-clock TNs, such as the parameterized versions of the protocols in $\left[\mathrm{BGK}^{+96]}\right.$ and [MT01]. This means that, despite our undecidability result, it is interesting to design semi-algorithms for multi-clock TNs. One direction for future work is to design acceleration techniques which are suffi ciently powerful to handle such classes of protocols.

\section{References}

[AAB00] A. Annichini, E. Asarin, and A. Bouajjani. Symbolic techniques for parametric reasoning about counter and clock systems. In Proc. CAV'O0, volume 1855 of $L N C S$, 2000.

[AČJYK00] Parosh Aziz Abdulla, Karlis Čer-ans, Bengt Jonsson, and Tsay Yih-Kuen. Algorithmic analysis of programs with well quasi-ordered domains. Information and Computation, 160:109-127, 2000.

[AD94] R. Alur and D. Dill. A theory of timed automata. Theoretical Computer Science, 126:183-235, 1994.

[AHV93] R. Alur, T. Henzinger, and M. Vardi. Parametric real-time reasoning. In Proc. $25^{\text {th }}$ ACM Symp. on Theory of Computing, LNCS, pages 592-601, 1993.

[AJ03] Parosh Aziz Abdulla and Bengt Jonsson. Model checking of systems with many identical timed processes. TCS, 290(1):241-264, 2003.

[AMC02] E. Asarin, O. Maler, and P. Caspi. Timed regular expressions. The Journal of ACM, 49(2):172-206, 2002. 
$\left[\mathrm{BGK}^{+}\right.$96] J. Bengtsson, W. O. D. Griffioen, K.J. Kristoffersen, K.G. Larsen, F. Larsson, P. Pettersson, and W. Yi. Verification of an audio protocol with bus collision using UPPAAL. In Proc. CAV'96, volume 1102 of LNCS, pages 244-256, 1996.

[Del00] G. Delzanno. Automatic verification of cache coherence protocols. In Proc. CAV'00, volume 1855 of $L N C S$, pages 53-68, 2000.

[Dic13] L. E. Dickson. Finiteness of the odd perfect and primitive abundant numbers with $n$ distinct prime factors. Amer. J. Math., 35:413-422, 1913.

[EFM99] J. Esparza, A. Finkel, and R. Mayr. On the verification of broadcast protocols. In Proc. LICS'99, 1999.

[EK03] E.A. Emerson and V. Kahlon. Model checking guarded protocols. In Proc. LICS'03, 2003.

[FRSB02] A. Finkel, J.-F. Raskin, M. Samuelides, and L. Van Begin. Monotonic extensions of petri nets: Forward and backward search revisited. In Proc. Infi nity'02, 2002.

[GS92] S. M. German and A. P. Sistla. Reasoning about systems with many processes. Journal of the ACM, 39(3):675-735, 1992.

[GW93] P. Godefroid and P. Wolper. Using partial orders for the efficient verification of deadlock freedom and safety properties. Formal Methods in System Design, 2(2):149164, 1993.

[JLL77] N. D. Jones, L. H. Landweber, and Y. E. Lyen. Complexity of some problems in Petri nets. Theoretical Computer Science, (4):277-299, 1977.

$\left[\mathrm{KLL}^{+}\right.$97] K.J. Kristoffersen, F. Larroussinie, K. G. Larsen, P. Pettersson, and W. Yi. A compositional proof of a real-time mutual exclusion protocol. In Proc. TAPSOFT '97, LNCS, 1997.

$\left[\mathrm{KMM}^{+}\right.$01] Y. Kesten, O. Maler, M. Marcus, A. Pnueli, and E. Shahar. Symbolic model checking with rich assertional languages. Theoretical Computer Science, 256:93-112, 2001.

[Min61] M. Minsky. Recursive unsolvabitity of post's problem of tag and other topics in the theory of turing machines. Ann. of Math., 74:437-455, 1961.

[MT01] I. A. Mason and C. L. Talcott. Simple network protocol simulation within maude. In ENTCS, volume 36. Elsevier, 2001.

[VW86] M. Y. Vardi and P. Wolper. An automata-theoretic approach to automatic program verification. In Proc. LICS'86, pages 332-344, 1986. 